\title{
A New Methodology to Establish Upper Bounds on Open-Cell Foams Homogenized Properties
}

\section{Z. Dimitrovová}

The methodology for determining the upper bounds on the homogenized linear elastic properties of cellular solids, described for the two-dimensional case in Dimitrovová and Faria (1999), is extended to three-dimensional open-cell foams. Besides the upper bounds, the methodology provides necessary and sufficient conditions on optimal media. These conditions are written in terms of generalized internal forces and geometrical parameters. In some cases dependence on internal forces can be replaced by geometrical expressions. In such cases, the optimality of some medium under consideration can be verified directly from the microstructure, without any additional calculation. Some of the bounds derived in this paper are published for the first time, along with a proof of their optimality.

Keywords. Upper bounds on effective properties, open-cell foams, homogenization techniques, energy methods, optimization, optimal microstructures.

\section{Introduction}

Cellular solids can either be found in nature or manufactured by foaming of polymers, metals and ceramics, or by other technologies, e.g. by CVD-chemical vapor deposition, or DMLS-Direct Metal Laser Sintering. They have a wide range of applications namely in absorbing the kinetic energy from impacts, or as thermal and electrical insulators. To exploit these properties fully and efficiently, suitable methodologies allowing a detailed characterization of the behavior of the cellular solids are needed. In this paper we will examine the upper bounds on homogenized linear elastic properties.

A cellular solid (a foam) is composed of an interconnected network of solid beams and shell parts, which can be assigned to cells that are repeated in the medium. Two essential features characterize cellular media:

- The size of the voids is very small compared to the size of the full medium, and thus homogenization techniques (see Duvaut (1976), Bensoussan at al. (1978), Suquet (1985), Bakhvalov and Panasenko (1989), Nemat-Nasser and Hori (1993)) can be used in determining of the effective properties.

- The relative density is low, usually below 0.3 (Gibson and Ashby (1988)). As a consequence, at least one dimension of the solid phase (thickness) at the cell level is small compared to the characteristic cell size. This condition justifies the use of structural theories in homogenization calculations instead of the full 3D elasticity model.

Cellular solids may be classified as closed-cell, partly open-cell and open-cell foams. In this work we will restrict our analysis to open-cell foams, which consist solely of solid beams. Then the name repetitive lattice structures can also be adopted.

Several works deal with the effective elastic properties of open-cell foams or repetitive lattice structures, but the upper bounds on them are rarely analyzed. The main monograph on cellular solids was published by Gibson and Ashby (1988). Extensive work by Christensen has been dedicated to the characterization of effectively isotropic open-cell microstructures, where the response is governed by bending or direct (axial) resistance, (Christensen (1994, 1995)). In Christensen (1995) the values of the upper bound on the effective bulk and shear modulus are presented. The value of the bulk modulus bound has also been addressed in several other works, but only in the sense of an effective property of some particular microstructure, (see e.g. Warren and Kraynik (1988, 1997), Kraynik and Warren (1994), Zhu et al. (1997)).

Methodologies for determining effective properties can be discrete or continuous. Discrete approaches are usually based on micromechanics. They exploit either the periodicity or the regularity of the medium under consideration. In the former case, the calculations are performed on a unit or a basic cell, while in the latter case either a representative volume element or a typical joint is used. For instance in Kraynik and Warren (1994) and Warren and Kraynik (1997) the effective properties are determined by considering a tetrahedral joint (Kelvin foam) under the assumption of affine displacements. Application of this methodology to a medium with randomly placed basic cells of the regular cubic lattice yields also the maximum shear response. In this context, we can also mention the work of Dimitrovová (1999), where there is a detailed discussion of applicability of the orientational averaging to periodic cells. Among other works, Grenestedt (1998) and Li, Gao and Roy (2003) should also be mentioned. Continuum modeling of repetitive lattice structures is reviewed by Noor (1988). The literature review in this paragraph is far from complete, because it is not the aim of this paper to determine homogenized properties, but their upper bounds.

The inverse problem of identifying microstructures that achieve the prescribed effective properties has also been extensively studied (see, e.g. Sigmund (1994), Neves et al. (2000), Gibiansky and Sigmund (2000), Guedes et al. (2003)). These methods exploit homogenization techniques, starting with a basic cell, whose shape must be specified in advance, and then the available material is optimally distributed within it. 
Cellular solids can be viewed as two-phase composites with void and solid (generally non-homogeneous) phases. Determining the bounds on composite effective properties has been the subject of considerable research for many years. It may be argued, that there is no need for a new methodology, since the bounds for foams can be obtained from the composite two-phase bounds, just by introducing zero void properties. This is true in $2 \mathrm{D}$, but in $3 \mathrm{D}$ the optimal foams must contain shell parts in some regimes of optimality (Allaire and Kohn (1993)), therefore upper bounds on the homogenized properties of open-cell foams are strictly lower than those for general foams, and the development of a new methodology addressing this issue is fully justified.

Only the upper bounds on effective elastic properties will be examined, because the lower bounds for media with one void phase are zero. Without loss of generality only open-cell foams with a periodic microstructure will be considered, because in a medium with a random microstructure, a representative volume element can be chosen so that a medium created by its periodic repetition will have the same effective properties as the original random one. The contribution of this paper is that it extends of the methodology proposed by Dimitrovová and Faria (1999) from 2D to 3D. The methodology is based on homogenization theory and does not require any restriction on the basic cell shape or arrangement. The influence of the boundary layer is not accounted for and it is assumed that the basic cell contains a finite number of structural members, i.e. beams or bars. The upper bounds are derived by a bounding procedure using results from linear algebra and the Voigt bound basic assumption (Hill (1963)). The main advantage of the new methodology is that the necessary and sufficient conditions characterizing the optimal media will immediately follow from the bounding procedure. These conditions are written in terms of generalized internal forces and geometrical parameters. The proposed methodology recovers the well known bounds for effectively isotropic open-cell foams, though with a different proof. The main contribution lies in identifying of new bounds on the effective shear moduli of open-cell microstructures with effective cubic symmetry. In such cases, dependence on internal forces in maximality conditions can be replaced by geometrical expressions, implying that the optimality of the medium under consideration can be verified directly from the microstructure, without any additional calculation. The approximations inherent to the methodology are within the structural simplifications commonly used. The limitations are based on the assumption of a finite number of structural members in the basic cell, allowing only the identification of single scale microstructures, which implicitly excludes multiple rank laminates (see e.g. Allaire and Aubry (1999)) and the Hashin spheres medium (Hashin (1962)).

The paper is organized as follows. The methodology is reviewed in Section 2, namely in Section 2.1 simplified assumptions and basic relations are introduced, in Section 2.2 it is shown that the optimal media can be initially searched within a specific class of micro-trusses (this term will be explained later on), and in Section 2.3 the methodology is reviewed within this restricted class. The bounds are proven in Section 3, along with a specification of the optimal media microstructures. The paper is concluded in Section 4 with a discussion and an analysis of the developments.

\section{Review of the new methodology}

\section{1 Simplifying assumptions and basic relations}

The basic cell, $\vartheta$, defined as the (smallest) region of a periodic medium that can compose the full one by periodic repetition, will be conveniently rescaled to $\mathrm{V}$, where the spatial microvariable $\mathbf{y}$ is introduced. It is assumed that $\mathrm{V}$ contains a finite number of beams and that the solid phase is homogeneous and isotropic. Therefore the term material volume fraction can be used instead of relative density.

There are two extreme possibilities for the structural model of a joint between the beams composing the foam: (i) a pin joint and (ii) a rigid joint. A pin joint cannot transmit bending moments, and therefore it allows rotations of the structural members connected to it. Consequently, a non-loaded structural member with two pin joints can only support the internal forces acting in the direction of the line connecting the joints. On the other hand, a rigid joint preserves the angles between the beams connected to it. If all joints are rigid, the term micro-frame medium can be used; on the other hand not necessarily straight structural members connected by pin joints will be named as micro-truss media. Therefore any micro-frame medium has its related micro-truss, which is obtained by switching the behavior of rigid joints to pin joints. In reality, joint behavior is somewhere between these two extreme cases and should be represented by a flexible joint. Pin joint behavior can be achieved either by special construction allowing for rotations of the connected members or as a limit case: if the beams connected to a given joint have uniform cross sectional areas and the material volume fraction tends to zero, then the flexible joint approaches pin joint behavior.

In structural theories, beams are defined by their middle axes and joints can be replaced by single points (joint "centers") located in the intersection of the middle axes. The term theoretical length will be used to identify the middle axis length between the joint centers, and active length will be usedto identify the same length shortened by the parts inside the joints (Fig. 1). Small discrepancies when middle axes do not intersect exactly at a single point will not be considered.

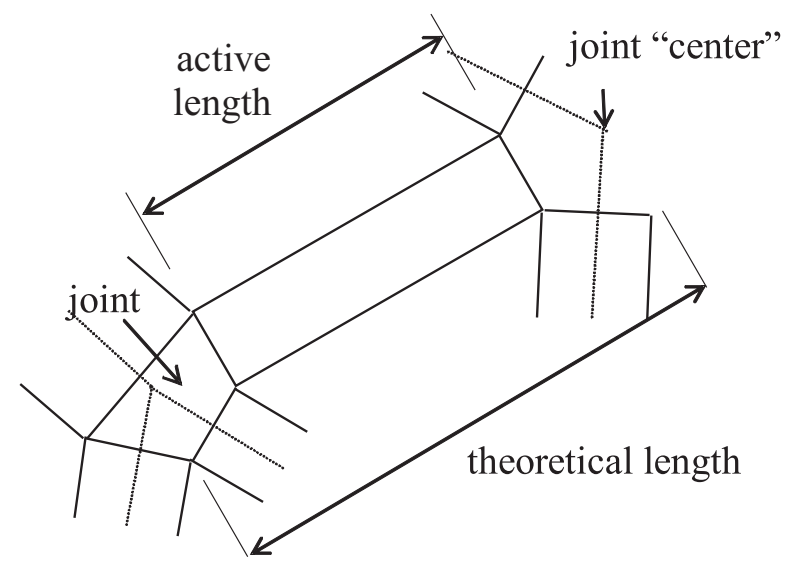

Fig. 1: Introduction of theoretical and active lengths 
We will address only open-cell foams with effective isotropy or cubic symmetry. The tensor of the effective elastic constants can thus be written in dimensionless matrix form as:

$$
\mathbf{C}^{*}=\left[\begin{array}{cc}
\mathbf{C}_{1}^{*} & \mathbf{0} \\
\mathbf{0} & \mathbf{C}_{2}^{*}
\end{array}\right],
$$

where $\mathbf{C}_{2}^{*}=\mathrm{G}_{2}^{*} \mathbf{I}$ and

$$
\mathbf{G}_{1}^{*}=\left[\begin{array}{ccc}
\mathrm{K}^{*}+4 \mathrm{G}_{1}^{*} / 3 & \mathrm{~K}^{*}-2 \mathrm{G}_{1}^{*} / 3 & \mathrm{~K}^{*}-2 \mathrm{G}_{1}^{*} / 3 \\
& \mathrm{~K}^{*}+4 \mathrm{G}_{1}^{*} / 3 & \mathrm{~K}^{*}-2 \mathrm{G}_{1}^{*} / 3 \\
\text { symm. } & & \mathrm{K}^{*}+4 \mathrm{G}_{1}^{*} / 3
\end{array}\right] .
$$

Here $\mathbf{I}$ stands for the unit 3 by 3 matrix and $\mathbf{0}$ for the zero 3 by 3 matrix. Effective engineering constants $K, G_{1}$ and $G_{2}$ are the homogenized bulk and two shear moduli, respectively. Their dimensionless values with respect to the solid phase Young's modulus $E_{s}$ are identified as: $\mathrm{K}^{*}=\mathrm{K} / \mathrm{E}_{\mathrm{s}}, \mathrm{G}_{1}^{*}=\mathrm{G}_{1} / \mathrm{E}_{\mathrm{s}}$ and $G_{2}^{*}=G_{2} / E_{s}$. A medium is effectively isotropic when $G_{1}^{*}=G_{2}^{*}=G^{*}$. The above matrix form of the fourth order tensor of elastic constants (Lekhnitskii (1981)) in terms of the engineering constants $\mathrm{K}^{*}, \mathrm{G}_{1}^{*}$ and $\mathrm{G}_{2}^{*}$ is presented in Hashin and Shtrikman (1962).

At first, the aim is to determine each of the macroscopic engineering constants in terms of the generalized internal forces, which will form the initial relation for the bounding procedure. The global strain energy density W can be expressed for isotropic media as:

$$
\mathrm{W}=\frac{1}{2 \mathrm{E}_{\mathrm{S}}}\left(\frac{\Sigma_{\mathrm{M}}^{2}}{\mathrm{~K}^{*}}+\frac{\Sigma_{\mathrm{D}}: \Sigma_{\mathrm{D}}}{2 \mathrm{G}^{*}}\right)
$$

and for media with effective cubic symmetry as:

$\mathrm{W}=\frac{1}{2 \mathrm{E}_{\mathrm{s}}}\left(\frac{\Sigma_{\mathrm{M}}^{2}}{\mathrm{~K}^{*}}+\frac{\Sigma_{\mathrm{D}, 12}^{2}+\Sigma_{\mathrm{D}, 13}^{2}+\Sigma_{\mathrm{D}, 23}^{2}}{\mathrm{G}_{2}^{*}}+\right.$

$\left.+\frac{\left(\Sigma_{\mathrm{D}, 11}-\Sigma_{\mathrm{D}, 22}\right)^{2}+\left(\Sigma_{\mathrm{D}, 11}-\Sigma_{\mathrm{D}, 33}\right)^{2}+\left(\Sigma_{\mathrm{D}, 22}-\Sigma_{\mathrm{D}, 33}\right)^{2}}{6 \mathrm{G}_{1}^{*}}\right)$,

where $\Sigma_{\mathrm{M}}$ and $\Sigma_{\mathrm{D}}$ are the volumetric and deviatoric parts of the global stress tensor $\Sigma$ and $\Sigma_{\mathrm{D}}: \Sigma_{\mathrm{D}}=\Sigma_{\mathrm{D}, \mathrm{ij}} \Sigma_{\mathrm{D}, \mathrm{ij}}$ (the summation convention is adopted). The test macroloads to be applied on the medium and consequently on the basic cell can be chosen so that only one effective engineering constant will be left in (2) or (3), and can thus be expressed independently of the others and in terms of $\Sigma$ components and W. Examples of these macroloads are specified in Table 1. It is seen that the corresponding macrostrain $\mathbf{E}$ must fulfill similar conditions. Macrostrain $\mathbf{E}$ is connected to Macrostress $\Sigma$ by $\Sigma=\mathrm{E}_{\mathrm{s}} \mathbf{C}^{*} \cdot \mathbf{E}$, where

$\Sigma=\left\{\Sigma_{11}, \Sigma_{22}, \Sigma_{33}, \Sigma_{23}, \Sigma_{31}, \Sigma_{12}\right\}^{\mathrm{T}}$,

$\mathbf{E}=\left\{E_{11}, E_{22}, E_{33}, 2 E_{23}, 2 E_{31}, 2 E_{12}\right\}^{\mathrm{T}}$

and "." stands for matrix multiplication.

$\Sigma$ and W can be expressed with the help of an averaging operator applied on the local characteristics, $\sigma$ and w, (Suquet (1985)):

$\Sigma_{\mathrm{jk}}=\frac{1}{|\mathrm{~V}|} \int_{V^{\#}} \sigma_{\mathrm{jk}} \mathrm{d} \mathbf{y}=\frac{1}{|\mathrm{~V}|} \sum_{i} \int_{V^{\#} i} \sigma_{j \mathrm{j}}^{\mathrm{i}} \mathrm{d} \mathbf{y}=\sum_{\mathrm{i}}\left\langle\sigma_{\mathrm{jk}}^{\mathrm{i}}\right\rangle$,

$\mathrm{W}=\frac{1}{|\mathrm{~V}|} \int_{V^{\#}} \mathrm{w} \mathrm{d} \mathbf{y}=\frac{1}{|\mathrm{~V}|} \sum_{i} \int_{V^{\#} i} \mathrm{w}^{\mathrm{i}} \mathrm{d} \mathbf{y}=\sum_{\mathrm{i}}\left\langle\mathrm{w}^{\mathrm{i}}\right\rangle$,

where $\sigma^{\mathrm{i}}$ and $\mathrm{w}^{\mathrm{i}}$ are the local stress and the local strain energy density corresponding to the $\mathrm{i}^{\text {th }}$ beam (i-beam). The volume of the full cell is $|\mathrm{V}|$ while the volume of the i-beam is $\left|V_{i}^{\#}\right| \cdot \mid V_{i}^{\#}$ is composed of the volume corresponding to the active length plus the corresponding volume in the connected parts within the joints, so that $\left|\mathrm{V}_{\mathrm{i}}^{\#} \cap \mathrm{V}_{\mathrm{j}}^{\#}\right|=0 \forall \mathrm{i} \neq \mathrm{j}$ and $\sum_{\mathrm{i}}\left|\mathrm{V}_{\mathrm{i}}^{\#}\right|=\left|\mathrm{V}^{\#}\right|$. $\mathrm{V}^{\#}$ is the volume of the material part in the cell. Due to the periodic repetition it is not necessary to treat separately the case when the i-beam is cut by the boundary of the cell.

Next, it is necessary to express the contributions of each i-beam, $\left\langle\sigma^{i}\right\rangle$ and $\left\langle w^{i}\right\rangle$, in terms of generalized internal forces. Looking at $\left\langle\sigma^{i}\right\rangle$, the formula from Nemat-Nasser and Hori (1993)

$\left\langle\sigma_{m j}^{i}\right\rangle=\frac{1}{\left|V_{i}^{\#}\right|} \int_{\partial V_{i}^{\#}} \sigma_{j k}^{i} n_{k} b_{m} d \mathbf{S}=\frac{1}{\left|V_{i}^{\#}\right|} \int_{\partial V_{i}^{\#}} t_{j}^{i} b_{m} d \mathbf{S}$

\begin{tabular}{|c|c|c|c|}
\hline Macroload & Property & Specification of $\Sigma$ & Specification of $\mathbf{E}$ \\
\hline$\Sigma^{\mathrm{K}}$ & $\mathrm{K}^{*}$ & $\begin{array}{c}\Sigma=\Sigma_{11}=\Sigma_{22}=\Sigma_{33} \neq 0 \\
\Sigma_{\mathrm{ij}}=0 \forall \mathrm{i} \neq \mathrm{j}\end{array}$ & $\begin{array}{c}\mathrm{E}=\mathrm{E}_{11}=\mathrm{E}_{22}=\mathrm{E}_{33} \neq 0 \\
\mathrm{E}_{\mathrm{ij}}=0 \forall \mathrm{i} \neq \mathrm{j}, 3 \mathrm{~K}^{*}=\Sigma /\left(\mathrm{EE}_{\mathrm{s}}\right)\end{array}$ \\
\hline$\Sigma^{1 G}$ & $\mathrm{G}_{1}^{*}$ & $\begin{array}{c}\Sigma_{11}+\Sigma_{22}+\Sigma_{33}=0, \exists \mathrm{k} ; \Sigma_{\mathrm{kk}} \neq 0 \\
\Sigma_{\mathrm{ij}}=0 \forall \mathrm{i} \neq \mathrm{j}\end{array}$ & $\begin{aligned} \mathrm{E}_{11}+\mathrm{E}_{22}+\mathrm{E}_{33} & =0, \mathrm{E}_{\mathrm{ij}}=0 \quad \forall \mathrm{i} \neq \mathrm{j} \\
2^{1} \mathrm{G}^{*} & =\Sigma_{\mathrm{kk}} /\left(\mathrm{E}_{\mathrm{kk}} \mathrm{E}_{\mathrm{s}}\right) \forall \mathrm{k}^{\#}\end{aligned}$ \\
\hline$\Sigma^{2 \mathrm{G}}$ & $\mathrm{G}_{2}^{*}$ & $\Sigma_{11}=\Sigma_{22}=\Sigma_{33}=0, \exists \mathrm{i} \neq \mathrm{j} ; \Sigma_{\mathrm{ij}} \neq 0$ & $\begin{array}{c}\mathrm{E}_{11}=\mathrm{E}_{22}=\mathrm{E}_{33}=0, \\
2^{2} \mathrm{G}^{*}=\Sigma_{\mathrm{ij}} /\left(\mathrm{E}_{\mathrm{ij}} \mathrm{E}_{\mathrm{s}}\right) \forall \mathrm{i} \neq \mathrm{j}^{\#}\end{array}$ \\
\hline$\Sigma^{\mathrm{G}}$ & $\mathrm{G}^{*}$ & $\begin{array}{c}\Sigma_{11}+\Sigma_{22}+\Sigma_{33}=0, \exists \mathrm{k} ; \Sigma_{\mathrm{kk}} \neq 0, \\
\exists \mathrm{i} \neq \mathrm{j} ; \Sigma_{\mathrm{ij}} \neq 0\end{array}$ & $\begin{array}{c}\mathrm{E}_{11}=\mathrm{E}_{22}=\mathrm{E}_{33}=0, \\
2 \mathrm{G}^{*}=\Sigma_{\mathrm{ij}} /\left(\mathrm{E}_{\mathrm{ij}} \mathrm{E}_{\mathrm{s}}\right) \forall \mathrm{i}, \mathrm{j}^{\#}\end{array}$ \\
\hline
\end{tabular}

Table 1: Test macroloads and the corresponding specification of the macrostress and the macrostrain

(\# if the macrostress component is different from zero) 
can be exploited. In (6), $\mathbf{b}$ is the position vector of the points on $\partial \mathrm{V}_{\mathrm{i}}^{\#}$ and $\mathbf{t}$ is the boundary traction. If $\mathbf{t}$ is self-equilibrated, then $\left\langle\sigma^{i}\right\rangle$ is symmetric and the integral in (6) does not depend on the origin of the coordinate system for $\mathbf{b}$. The expression for $\left\langle w^{i}\right\rangle$ can be, as usual, simplified by considering that generalized internal forces act over theoretical lengths and that the contribution of the joints is negligible.

\subsection{Micro-trusses with straight bars of constant cross sectional area versus micro-frames}

Optimal low-density micro-frame open-cell foams will be defined as those for which the related micro-truss is optimal. Justification of this definition and more details on optimal micro-trusses are presented in this subsection, namely it will be proven that optimal micro-trusses can only be composed of straight bars with a constant cross section.

In order to justify the definition stated above, it is necessary to verify that a curved beam cannot from part of the optimal low-density media. Let us suppose that the i-beam of a micro-frame basic cell is curved. Then a local coordinate system $\left(z_{1}, z_{2}\right)$ can be introduced so that $z_{1}$ connects the centers of the joints (Fig. 2). The middle axis of the beam is given by $\mathrm{z}_{2}=\mathrm{a}\left(\mathrm{z}_{1}\right)$ and $\mathrm{r}$ designates the curved coordinate. Let us separate the beam of active length from the joints by the cuts shown in Fig. 2. It is assumed that there exists a plane containing the i-beam middle curve and that the macroload acts in such a way that the generalized internal forces in the beam cuts are also contained in this plane. The geometrical param- eters $\alpha(r), \alpha_{0 k}, \alpha_{0 m}, h_{k}, h_{m}, v_{k}, v_{m}, l, p$, the generalized internal forces in the beam cuts F, B and D and other local auxiliary coordinate systems $\left(\widetilde{z}_{1}, \widetilde{z}_{2}\right)$ and $\left(\hat{z}_{1}, \hat{z}_{2}\right)$ are specified in Fig. $2 . l$ and $\mathrm{p}$ are projections of the theoretical and the active lengths on $\mathrm{z}_{1}$ and the bending moment along the beam is separated into its (average) constant (D) and "antisymmetric" parts.

For the i-beam let us express the average quantities $\left\langle\sigma^{\mathrm{i}}\right\rangle$ and $\left\langle w^{i}\right\rangle$ in terms of the generalized internal forces and discuss the possibility of its position in an optimal medium. Superscript " $i$ " will be omitted for the sake of simplicity, whenever no confusion is possible. It must be pointed out that local stress averaging cannot be performed over the theoretical length, because this would cause overlapping of the joints. Thus the i-beam average stress $\langle\sigma\rangle$ must be expressed as

$$
\langle\sigma\rangle=\left\langle\sigma_{\mathrm{bm}}\right\rangle+\left\langle\sigma_{\mathrm{jk}}\right\rangle+\left\langle\sigma_{\mathrm{jm}}\right\rangle,
$$

where the contribution with subscript "bm" relates to the beam with active length and thoses with "jk" and "jm" subscripts relate to the left $(\mathrm{k})$ and right $(\mathrm{m})$ adjacent joint parts, respectively. Strict application of (6), in the previous expression, would imply integration over the internal faces of the joints, which is complicated. To overcome these difficulties we can define

$$
\langle\widetilde{\sigma}\rangle=\left\langle\sigma_{\mathrm{bm}}\right\rangle+\left\langle\widetilde{\sigma}_{\mathrm{jk}}\right\rangle+\left\langle\widetilde{\sigma}_{\mathrm{jm}}\right\rangle,
$$

where $\left\langle\widetilde{\sigma}_{j k}\right\rangle$ and $\left\langle\widetilde{\sigma}_{j m}\right\rangle$ stand only for the contribution of the faces where the beam was cut. Then $\left\langle\widetilde{\sigma}_{j k}\right\rangle$ and $\left\langle\widetilde{\sigma}_{j m}\right\rangle$ are coordinate system dependent and therefore their coordinate

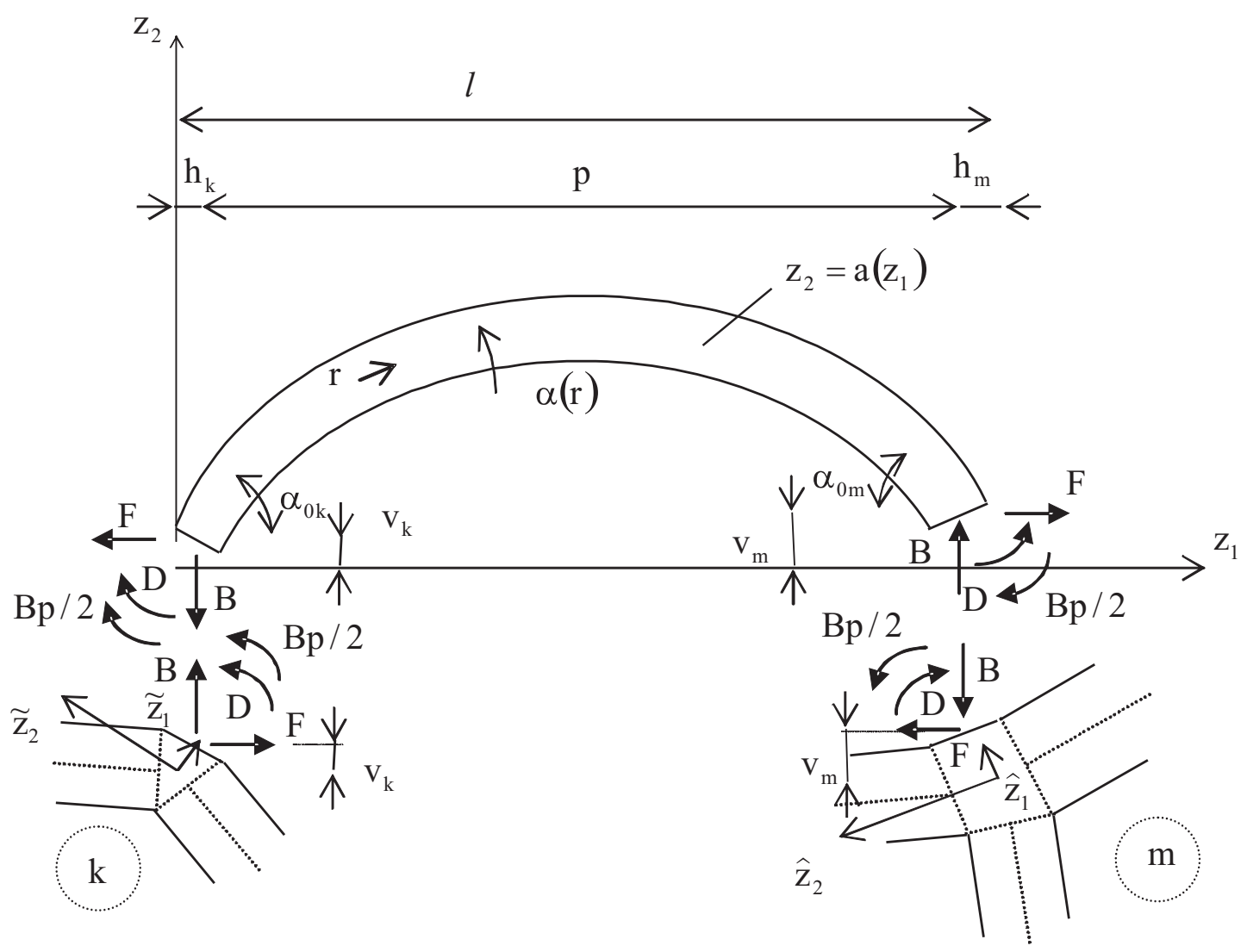

Fig. 2: Specification of the curved i-beam 
systems must be uniquely defined in a way applicable to any beam from the basic cell. Coordinate systems $\left(\widetilde{z}_{1}, \widetilde{z}_{2}\right)$ and $\left(\hat{z}_{1}, \hat{z}_{2}\right)$ are introduced as specified in Fig. 2. With respect to $\left(\mathrm{z}_{1}, \mathrm{z}_{2}\right)$ this yields from (6):

$$
|\mathrm{V}|\left\langle\sigma_{\mathrm{bm}}\right\rangle=\left[\begin{array}{cc}
\mathrm{Fp} & \frac{1}{2} \mathrm{Bp}\left(\cos ^{2} \alpha_{0 \mathrm{k}}+\cos ^{2} \alpha_{0 \mathrm{~m}}\right) \\
-\frac{1}{2} \mathrm{~B}\left(\sin \alpha_{0 \mathrm{k}} \cos \alpha_{0 \mathrm{k}}-\sin \alpha_{0 \mathrm{~m}} \cos \alpha_{0 \mathrm{~m}}\right) & +\mathrm{D}\left(\cos ^{2} \alpha_{0 \mathrm{k}}-\cos ^{2} \alpha_{0 \mathrm{~m}}\right) \\
-\mathrm{D}\left(\sin \alpha_{0 \mathrm{k}} \cos \alpha_{0 \mathrm{k}}+\sin \alpha_{0 \mathrm{~m}} \cos \alpha_{0 \mathrm{~m}}\right) & \\
-\mathrm{F}\left(\mathrm{v}_{\mathrm{k}}-\mathrm{v}_{\mathrm{m}}\right) & \frac{1}{2} \mathrm{Bp}\left(\sin \alpha_{0 \mathrm{k}} \cos \alpha_{0 \mathrm{k}}-\sin \alpha_{0 \mathrm{~m}} \cos \alpha_{0 \mathrm{~m}}\right) \\
+\frac{1}{2} \mathrm{Bp}\left(\cos ^{2} \alpha_{0 \mathrm{k}}+\cos ^{2} \alpha_{0 \mathrm{~m}}\right) & +\mathrm{B}\left(\mathrm{v}_{\mathrm{k}}-\mathrm{v}_{\mathrm{m}}\right) \\
+\mathrm{D}\left(\cos ^{2} \alpha_{0 \mathrm{k}}-\cos ^{2} \alpha_{0 \mathrm{~m}}\right) & +\mathrm{D}\left(\sin \alpha_{0 \mathrm{k}} \cos \alpha_{0 \mathrm{k}}+\sin \alpha_{0 \mathrm{~m}} \cos \alpha_{0 \mathrm{~m}}\right)
\end{array}\right]
$$

With respect to $\left(\widetilde{\mathrm{z}}_{1}, \widetilde{\mathrm{z}}_{2}\right)$ and $\left(\hat{\mathrm{z}}_{1}, \hat{\mathrm{z}}_{2}\right)$ we can obtain:

$$
|\mathrm{V}|\left\langle\widetilde{\sigma}_{\mathrm{jk}}^{\prime}\right\rangle=\left[\begin{array}{cc}
\mathrm{Fh}_{\mathrm{k}}+\mathrm{Bv}_{\mathrm{k}} & -\mathrm{Fv}_{\mathrm{k}}+\mathrm{Bh}_{\mathrm{k}} \\
-\mathrm{Bp} / 2-\mathrm{D} & 0
\end{array}\right] \text { and }|\mathrm{V}|\left\langle\widetilde{\sigma}_{\mathrm{jm}}^{\prime}\right\rangle=\left[\begin{array}{cc}
\mathrm{Fh}_{\mathrm{m}}-\mathrm{Bv} \mathrm{v}_{\mathrm{m}} & \mathrm{Fv} \mathrm{v}_{\mathrm{m}}+\mathrm{Bh}_{\mathrm{m}} \\
-\mathrm{Bp} / 2+\mathrm{D} & 0
\end{array}\right] \text {, }
$$

which after rotation to $\left(\mathrm{z}_{1}, \mathrm{z}_{2}\right)$ yields:

$$
\begin{aligned}
\mathrm{V} \mid\left\langle\widetilde{\sigma}_{\mathrm{jk}}^{\prime}\right\rangle & =\left[\begin{array}{cc}
\mathrm{Fh}_{\mathrm{k}}+\frac{1}{2} \mathrm{Bp} \cos \alpha_{0 \mathrm{k}} \sin \alpha_{0 \mathrm{k}} & \mathrm{Bh}_{\mathrm{k}}+\frac{1}{2} \mathrm{Bp} \sin ^{2} \alpha_{0 \mathrm{k}} \\
+\mathrm{D} \cos \alpha_{0 \mathrm{k}} \sin \alpha_{0 \mathrm{k}} & +\mathrm{D} \sin ^{2} \alpha_{0 \mathrm{k}} \\
\mathrm{Fv}_{\mathrm{k}}-\frac{1}{2} \mathrm{Bp} \cos ^{2} \alpha_{0 \mathrm{k}} & \mathrm{Bv}_{\mathrm{k}}-\frac{1}{2} \mathrm{Bp} \cos \alpha_{0 \mathrm{k}} \sin \alpha_{0 \mathrm{k}} \\
-\mathrm{D} \cos ^{2} \alpha_{0 \mathrm{k}} & -\mathrm{D} \cos \alpha_{0 \mathrm{k}} \sin \alpha_{0 \mathrm{k}}
\end{array}\right] \text { and } \\
|\mathrm{V}|\left\langle\widetilde{\sigma}_{\mathrm{jm}}^{\prime}\right\rangle & {\left[\begin{array}{cc}
\mathrm{Fh}_{\mathrm{m}}-\frac{1}{2} \mathrm{Bp} \cos \alpha_{0 \mathrm{~m}} \sin \alpha_{0 \mathrm{~m}} & \mathrm{Bh}_{\mathrm{m}}+\frac{1}{2} \mathrm{Bp} \sin ^{2} \alpha_{0 \mathrm{~m}} \\
+\mathrm{D} \cos \alpha_{0 \mathrm{~m}} \sin \alpha_{0 \mathrm{~m}} & -\mathrm{D} \sin { }^{2} \alpha_{0 \mathrm{~m}} \\
-\mathrm{Fv}_{\mathrm{m}}-\frac{1}{2} \mathrm{Bp} \cos ^{2} \alpha_{0 \mathrm{~m}} & -\mathrm{Bv}_{\mathrm{m}}+\frac{1}{2} \mathrm{Bp} \cos \alpha_{0 \mathrm{~m}} \sin \alpha_{0 \mathrm{~m}} \\
+\mathrm{D} \cos { }^{2} \alpha_{0 \mathrm{~m}} & -\mathrm{D} \cos \alpha_{0 \mathrm{~m}} \sin \alpha_{0 \mathrm{~m}}
\end{array}\right], }
\end{aligned}
$$

which finally gives

$$
\langle\widetilde{\sigma}\rangle=\frac{l}{|\mathrm{~V}|}\left[\begin{array}{cc}
\mathrm{F} & \mathrm{B} \\
0 & 0
\end{array}\right]
$$

Origins of the coordinate systems $\left(\mathrm{z}_{1}, \mathrm{z}_{2}\right)$ and $\left(\widetilde{\mathrm{z}}_{1}, \widetilde{\mathrm{z}}_{2}\right)$ are coincident, therefore only the face of joint $(\mathrm{m})$ and the corresponding face of the beam can be considered to obtain (10). It is necessary to point out that the reason for non-symmetry of $\langle\widetilde{\sigma}\rangle$ is the omission of the contributions of the internal faces of the joints in (8-9), as explained above. This does not mean any inaccuracy, because after rotation of the contributions of all beams to the cell coordinate system and after summation over all the beams, the final expression for $\Sigma$ will be complete and symmetric.

When a general curved beam under a general macroload is considered, the local coordinate system $\left(\mathrm{z}_{1}, \mathrm{z}_{2}\right)$ connecting the centers of the jointscan also be introduced. Then it is necessary to replace internal force $\mathrm{B}$ by $\mathrm{B}_{1}$ and $\mathrm{B}_{2}$, bending moment $\mathrm{D}$ by $\mathrm{D}_{1}$ and $\mathrm{D}_{2}$, and to introduce torsion moment $\mathrm{T}$. Following the same procedure as above, we obtain:

$$
\langle\widetilde{\sigma}\rangle=\frac{l}{|\mathrm{~V}|}\left[\begin{array}{ccc}
\mathrm{F} & \mathrm{B}_{1} & \mathrm{~B}_{2} \\
0 & 0 & 0 \\
0 & 0 & 0
\end{array}\right] .
$$

It is important to realize that (11) has the same form as it would have for a related straight beam of theoretical length $l$, arbitrary cross sectional area variation and with the same generalized internal forces in the cuts. Therefore there is no distinction between the $\langle\sigma\rangle$ contribution of a straight or a curved beam to $\Sigma$. Moreover, (11) includes neither include the constant part of bending moments $D_{1}$ and $D_{2}$ nor the torsion moment $\mathrm{T}$. If the $\mathrm{i}$-were to have pin joints, then from equilibrium $\mathrm{B}_{1}=\mathrm{B}_{2}=0$. We point outd that in order to express $\Sigma,(11)$ should be rotated to the basic cell coordinates and summed over all the beams.

Now the average $\langle w\rangle$ will be determined. For the sake of simplicity it is again firstly assumed that a curved beam and the generalized forces are contained in a plane. As usual, the strain energy density corresponding to the shear forces can be omitted. Then we can write:

$$
\langle w\rangle=\frac{1}{2|V| E_{s}}\left(\int_{(a)} \frac{N^{2}(r)}{A(r)} d r+\int_{(a)} \frac{M^{2}(r)}{I(r)} d r\right)
$$


where $\mathrm{A}(\mathrm{r})$ and $\mathrm{I}(\mathrm{r})$ stand for cross sectional area and moment of inertia, respectively, $\mathrm{N}(\mathrm{r})$ and $\mathrm{M}(\mathrm{r})$ are normal forces and bending moments and (a) stands for the integration along the curved theoretical length of the beam. It may be pointed out that using theoretical lengths and overlapping in joints is an allowable and common simplification in strain energy. In accordance with this approximation, the originally introduced generalized forces F, B and D in the beam cuts do not have to change.

A cross sectional area $A_{0}$ of a related straight beam with constant cross section and the same volume as the original curved beam, can be introduced by (overlapping in the junctions can also be neglected here):

$$
\mathrm{A}_{0} l=\int_{0}^{l} \mathrm{~A}\left(\mathrm{z}_{1}\right) \sqrt{1+\left(\mathrm{a}^{\prime}\left(\mathrm{z}_{1}\right)\right)^{2}} \mathrm{~d} z_{1},
$$

where $\mathrm{a}^{\prime}\left(\mathrm{z}_{1}\right)=\frac{\mathrm{da}\left(\mathrm{z}_{1}\right)}{\mathrm{dz}}$.

Because there is no distinction between the $\langle\sigma\rangle$ contribution of a straight or a curved beam to $\Sigma$, let us minimize $\langle w\rangle$ in order to discuss the position of the i-beam in an optimal medium. This minimization must be performed over all possible shapes $\mathrm{a}\left(\mathrm{z}_{1}\right)$ and volume distributions along the middle curve:

$$
\begin{aligned}
& 2 \mathrm{E}_{\mathrm{s}}|\mathrm{V}| \cdot \min _{\mathrm{a}\left(\mathrm{z}_{1}\right) ; \mathrm{A}(\mathrm{r}) ; \mathrm{I}(\mathrm{r})}\langle\mathrm{w}\rangle= \\
= & \min _{\mathrm{a}\left(\mathrm{z}_{1}\right) ; \mathrm{A}(\mathrm{r}) ; \mathrm{I}(\mathrm{r})}\left(\int_{(\mathrm{a})} \frac{\mathrm{N}^{2}(\mathrm{r})}{\mathrm{A}(\mathrm{r})} \mathrm{dr}+\int_{(\mathrm{a})} \frac{\mathrm{M}^{2}(\mathrm{r})}{\mathrm{I}(\mathrm{r})} \mathrm{dr}\right) \geq \\
\geq & \min _{\mathrm{a}\left(\mathrm{z}_{1}\right) ; \mathrm{A}(\mathrm{r})} \int_{(\mathrm{a})} \frac{\mathrm{N}^{2}(\mathrm{r})}{\mathrm{A}(\mathrm{r})} \mathrm{dr}+\min _{\mathrm{a}\left(\mathrm{z}_{1}\right) ; \mathrm{I}(\mathrm{r})} \int_{(\mathrm{a})} \frac{\mathrm{M}^{2}(\mathrm{r})}{\mathrm{I}(\mathrm{r})} \mathrm{dr} .
\end{aligned}
$$

Equality in (14) can be achieved, if the minimizing shape and volume distribution are the same for both terms in the last part of (14).

The distribution of the normal forces can be written as:

$$
\mathrm{N}(\mathrm{r})=\mathrm{F} \cos \alpha(\mathrm{r})+\mathrm{B} \sin \alpha(\mathrm{r}) .
$$

Therefore:

$$
\int_{(\mathrm{a})} \frac{\mathrm{N}^{2}(\mathrm{r})}{\mathrm{A}(\mathrm{r})} \mathrm{dr}=\int_{0}^{l} \frac{\left(\mathrm{F}+\mathrm{Ba}^{\prime}\left(\mathrm{z}_{1}\right)\right)^{2}}{\mathrm{~A}\left(\mathrm{z}_{1}\right) \sqrt{1+\left(\mathrm{a}^{\prime}\left(\mathrm{z}_{1}\right)\right)^{2}}} \mathrm{dz} \text {. }
$$

Using the Schwarz inequality in the form of:

$$
\begin{aligned}
& \mathrm{F}^{2} l^{2}=\left(\int_{0}^{l}\left(\mathrm{~F}+\mathrm{Ba}^{\prime}\left(\mathrm{z}_{1}\right)\right) \mathrm{d} \mathrm{z}_{1}\right)^{2}= \\
& =\left(\int_{0}^{l} \frac{\mathrm{F}+\mathrm{Ba}^{\prime}\left(\mathrm{z}_{1}\right)}{\sqrt{\mathrm{A}\left(\mathrm{z}_{1}\right)} \sqrt[4]{1+\left(\mathrm{a}^{\prime}\left(\mathrm{z}_{1}\right)\right)^{2}}} \cdot\left(\sqrt{\mathrm{A}\left(\mathrm{z}_{1}\right)} \sqrt[4]{1+\left(\mathrm{a}^{\prime}\left(\mathrm{z}_{1}\right)\right)^{2}}\right) \mathrm{d} \mathrm{z}_{1}\right)^{2} \leq \\
& \leq\left(\int_{0}^{l} \frac{\left(\mathrm{F}+\mathrm{Ba}^{\prime}\left(\mathrm{z}_{1}\right)\right)^{2}}{\mathrm{~A}\left(\mathrm{z}_{1}\right) \sqrt{1+\left(\mathrm{a}^{\prime}\left(\mathrm{z}_{1}\right)\right)^{2}}} \mathrm{~d} \mathrm{z}_{1}\right) \cdot\left(\int_{0}^{l} \mathrm{~A}\left(\mathrm{z}_{1}\right) \sqrt{1+\left(\mathrm{a}^{\prime}\left(\mathrm{z}_{1}\right)\right)^{2}} \mathrm{~d} \mathrm{z}_{1}\right)= \\
& =\mathrm{A}_{0} l \int_{0}^{l} \frac{\left(\mathrm{F}+\mathrm{Ba}^{\prime}\left(\mathrm{z}_{1}\right)\right)^{2}}{\mathrm{~A}\left(\mathrm{z}_{1}\right) \sqrt{1+\left(\mathrm{a}^{\prime}\left(\mathrm{z}_{1}\right)\right)^{2}}} \mathrm{~d} \mathrm{z}_{1}
\end{aligned}
$$

gives the following inequality:

$$
\int_{0}^{l} \frac{\left(\mathrm{F}+\mathrm{Ba}^{\prime}\left(\mathrm{z}_{1}\right)\right)^{2}}{\mathrm{~A}\left(\mathrm{z}_{1}\right) \sqrt{1+\left(\mathrm{a}^{\prime}\left(\mathrm{z}_{1}\right)\right)^{2}}} \mathrm{dz} \mathrm{z}_{1} \geq \mathrm{F}^{2} \frac{l}{\mathrm{~A}_{0}} .
$$

Equality in (18 or 17) can only be achieved if

$$
\frac{\mathrm{F}+\mathrm{Ba}^{\prime}\left(\mathrm{z}_{1}\right)}{\mathrm{A}\left(\mathrm{z}_{1}\right) \sqrt{1+\left(\mathrm{a}^{\prime}\left(\mathrm{z}_{1}\right)\right)^{2}}}
$$

is constant with respect to $z_{1}$, which implies that the beam must be straight and with a constant cross sectional area. Then contribution of the normal forces to $\langle w\rangle$ does not include B.

The distribution of the bending moments can be expressed as:

$$
\mathrm{M}\left(\mathrm{z}_{1}\right)=\mathrm{D}+\mathrm{Fa}\left(\mathrm{z}_{1}\right)+\mathrm{B}\left(\frac{l}{2}-\mathrm{z}_{1}\right)
$$

When minimizing conditions for normal forces contribution to $\langle w\rangle$ are used, it is sufficient to look at

$$
\int_{0}^{l}\left(\mathrm{D}+\mathrm{B}\left(\frac{l}{2}-\mathrm{z}_{1}\right)\right)^{2} \mathrm{~d} \mathrm{z}_{1}=\mathrm{D}^{2} l+\mathrm{B}^{2} l^{3} / 12 .
$$

The optimal media require $\mathrm{D}=0$, because $\mathrm{D}$ does not appear in (10). If moreover $\mathrm{B}=0$, as a consequence of constant cross sectional area and the material volume fraction going to zero, then the contribution of the bending moments is zero and the last term in (14) reaches its trivial minimum. Extension of this statement to a general curved beam under a general macroload is clear, there would only be one more integral in the form of (19) and a separate T contribution, which can be required to be zero, because $\mathrm{T}$ does not appear in (11).

This justifies the definition of optimal media stated at the beginning of this subsection, and proves that optimal micro-trusses must be composed of straight bars with constant cross sectional areas. Nevertheless the contribution of bending is not excluded from the optimal media, when behaving as micro-frames.

In summary, optimal open-cell foams can be searched within the class of micro-trusses with straight bars of constant cross section. In this class the bound can be expressed as a linear function of the material volume fraction, s, as shown in Dimitrovová and Faria (1999) and as clarified in Section 3. Related optimal micro-frames can develop non-zero bending moments, but only in their antisymmetric form (in terms of $\mathrm{B}_{1}$ and/or $\mathrm{B}_{2}$ ). If bending moments are presented, the corresponding effective engineering constant, written as a Taylor's expansion in s, contains a quadratic term (a detailed discussion is provided in Dimitrovová and Faria (1999)). The tangent at $s=0$, i.e. the linearized bound, relates to the same property of the corresponding micro-truss. Please note, however, (see Fig. 3) that for a particularly high material volume fraction, $\mathrm{s}_{0}$, there can exist a micro-frame with a higher elastic property than that which is obtained from the optimal micro-truss. These cases are of no interest here since for low-density media only the initial slope (linearized property) matters. For the same reasons media with only a bending response are strictly excluded from the class of optimal micro-frames, because the corresponding micro-truss is a kinematic mechanism and the linearized bound is zero. 


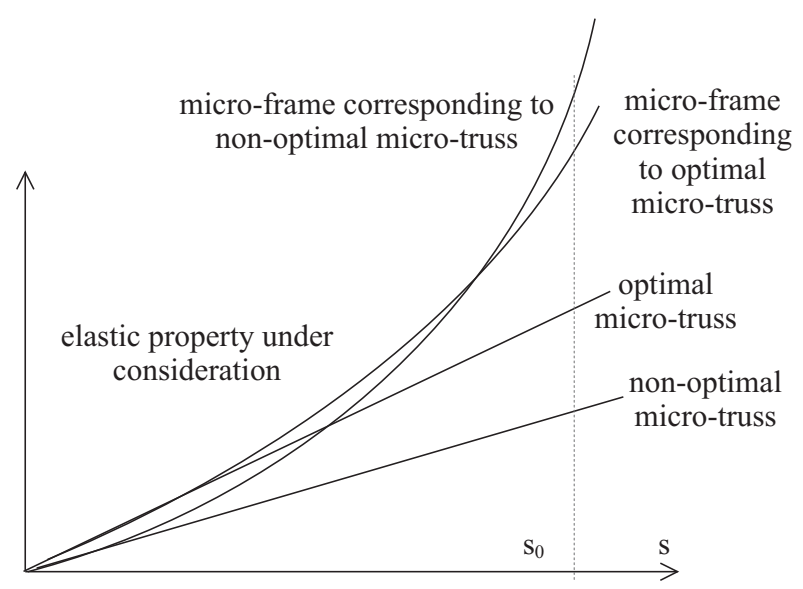

Fig. 3: Specification of optimal media response

It was shown in Dimitrovová and Faria (1999) that if the bulk modulus is under consideration, then the macrostress components that are necessary to express this property do not contain a contribution of $\mathrm{B}$. Then bending moments are excluded from optimal media, not only in the limit at $\mathrm{s}=0$, but in the full range of low-density s values. This result is readily extendable to 3D.

In Section 3.2, optimal micro-trusses for shear modulus $\mathrm{G}_{1}^{*}$ of media with effective cubic symmetry will be fully geometrically specified. In this case it will be seen that switching to micro-frames will not develop bending moments. So also here the upper bound is linear within the validity of structural theories. The bending contribution is present only in isotropic shear $\mathrm{G}^{*}$ and in $\mathrm{G}_{2}^{*}$.

\subsection{Review of the methodology in the class of micro-truss media with straight bars of constant cross section}

In the class of micro-trusses, the normal force is the only generalized internal force in the medium. Let an arbitrary basic cell consisting of $\mathrm{n}$ bars be assumed. The contributions $\left\langle\sigma^{i}\right\rangle$ and $\left\langle w^{i}\right\rangle$ of each i-bar of theoretical length $l_{i}$, cross sectional area $\mathrm{A}_{\mathrm{i}}$ and normal force $\mathrm{N}_{\mathrm{i}}$ can be specified in the following way (compare with (11)):

$\left\langle\sigma^{\mathrm{i}}\right\rangle=\frac{\mathrm{N}_{\mathrm{i}} l_{\mathrm{i}}}{|\mathrm{V}|}$.

$\left[\begin{array}{ccc}\cos ^{2} \varphi_{\mathrm{i}} \sin ^{2} \theta_{\mathrm{i}} ; & \sin \varphi_{\mathrm{i}} \cos \varphi_{\mathrm{i}} \sin ^{2} \theta_{\mathrm{i}} ; & \cos \varphi_{\mathrm{i}} \sin \theta_{\mathrm{i}} \cos \theta_{\mathrm{i}} \\ & \sin ^{2} \varphi_{\mathrm{i}} \sin ^{2} \theta_{\mathrm{i}} ; & \sin \varphi_{\mathrm{i}} \sin \theta_{\mathrm{i}} \cos \theta_{\mathrm{i}} \\ \text { symm. } & & \cos ^{2} \theta_{\mathrm{i}}\end{array}\right]$,

0)

where the two spherical angles $\theta_{i} \in\langle 0, \pi)$ and $\varphi_{i} \in\langle 0,2 \pi)$ specify the i-bar position with respect to the cell coordinates $\mathrm{y}_{\mathrm{j}}, \mathrm{j}=1,2,3$, (Fig. 4); and (see (14) and (18))

$$
\left\langle\mathrm{w}^{\mathrm{i}}\right\rangle=\frac{1}{2|\mathrm{~V}| \mathrm{E}_{\mathrm{s}}}\left(\mathrm{N}_{\mathrm{i}}^{2} \frac{\mathrm{l}_{\mathrm{i}}}{\mathrm{A}_{\mathrm{i}}}\right) .
$$

Let the following designation be introduced:

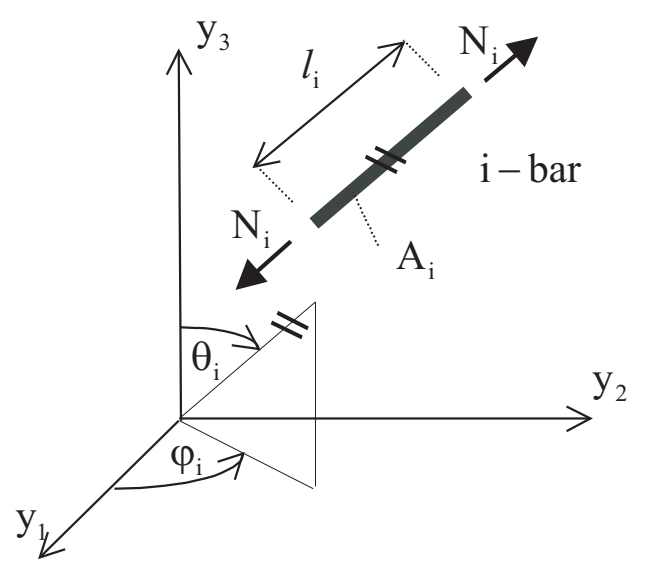

Fig. 4: Specification of the i-bar within the basic cell

$\Omega_{1, \mathrm{i}}=\cos ^{2} \varphi_{\mathrm{i}} \sin ^{2} \theta_{\mathrm{i}}$,

$\Omega_{2, \mathrm{i}}=\sin ^{2} \varphi_{\mathrm{i}} \sin ^{2} \theta_{\mathrm{i}}$,

$\Omega_{3, \mathrm{i}}=\cos ^{2} \theta_{\mathrm{i}}$;

$\Phi_{1, \mathrm{i}}=\sin \varphi_{\mathrm{i}} \sin \theta_{\mathrm{i}} \cos \theta_{\mathrm{i}}$,

$\Phi_{2, \mathrm{i}}=\cos \varphi_{\mathrm{i}} \sin \theta_{\mathrm{i}} \cos \theta_{\mathrm{i}}$,

$\Phi_{3, \mathrm{i}}=\sin \varphi_{\mathrm{i}} \cos \varphi_{\mathrm{i}} \sin ^{2} \theta_{\mathrm{i}}$

$\Psi_{1, \mathrm{i}}=\sin ^{2} \varphi_{\mathrm{i}} \sin ^{2} \theta_{\mathrm{i}}-\cos ^{2} \theta_{\mathrm{i}}$,

$\Psi_{2, \mathrm{i}}=\cos ^{2} \theta_{\mathrm{i}}-\cos ^{2} \varphi_{\mathrm{i}} \sin ^{2} \theta_{\mathrm{i}}$

$\Psi_{3, \mathrm{i}}=\cos \left(2 \varphi_{\mathrm{i}}\right) \sin ^{2} \theta_{\mathrm{i}}$;

then the vectors $\mathbf{N}, \mathbf{R}, \mathbf{Q}$ and $\mathbf{L}$ (compare with Dimitrovová and Faria (1999)) can be defined as:

$\mathbf{N}=\left\{\mathrm{N}_{1} \sqrt{\frac{l_{1}}{\mathrm{~A}_{1}}}, \mathrm{~N}_{2} \sqrt{\frac{l_{2}}{\mathrm{~A}_{2}}}, \ldots, \mathrm{N}_{\mathrm{n}} \sqrt{\frac{l_{\mathrm{n}}}{\mathrm{A}_{\mathrm{n}}}}\right\}$,

$\mathbf{R}_{\mathrm{j}}=\left\{\Omega_{\mathrm{j}, 1} \sqrt{\mathrm{l}_{1} \mathrm{~A}_{1}}, \Omega_{\mathrm{j}, 2} \sqrt{\mathrm{l}_{2} \mathrm{~A}_{2}}, \ldots, \Omega_{\mathrm{j}, \mathrm{n}} \sqrt{\mathrm{l}_{\mathrm{n}} \mathrm{A}_{\mathrm{n}}}\right\}, \mathrm{j}=1,2,3$,

$\mathbf{Q}_{j}=\left\{\Phi_{j, 1} \sqrt{l_{1} A_{1}}, \Phi_{j, 2} \sqrt{l_{2} A_{2}}, \ldots, \Phi_{j, n} \sqrt{l_{n} A_{n}}\right\}, j=1,2,3$,

$\mathbf{L}=\left\{\sqrt{l_{1} \mathrm{~A}_{1}}, \sqrt{l_{2} \mathrm{~A}_{2}}, \ldots, \sqrt{l_{\mathrm{n}} \mathrm{A}_{\mathrm{n}}}\right\}$

In addition, let us denote:

$\mathbf{P}_{1}=\mathbf{R}_{2}-\mathbf{R}_{3}, \mathbf{P}_{2}=\mathbf{R}_{3}-\mathbf{R}_{1}, \mathbf{P}_{3}=\mathbf{R}_{1}-\mathbf{R}_{2}$.

Thus:

$\mathbf{P}_{\mathrm{j}}=\left\{\Psi_{\mathrm{j}, 1} \sqrt{\mathrm{l}_{1} \mathrm{~A}_{1}}, \Psi_{\mathrm{j}, 2} \sqrt{\mathrm{l}_{2} \mathrm{~A}_{2}}, \ldots, \Psi_{\mathrm{j}, \mathrm{n}} \sqrt{\mathrm{l}_{\mathrm{n}} \mathrm{A}_{\mathrm{n}}},\right\}$,

$\mathrm{j}=1,2,3$

and it holds:

$\mathbf{P}_{1}+\mathbf{P}_{2}+\mathbf{P}_{3}=\mathbf{0}, \quad \mathbf{R}_{1}+\mathbf{R}_{2}+\mathbf{R}_{3}=\mathbf{L}$,

$\left\|\mathbf{P}_{1}\right\|^{2}+\left\|\mathbf{P}_{2}\right\|^{2}+\left\|\mathbf{P}_{3}\right\|^{2}+\left\|\mathbf{Q}_{1}\right\|^{2}+\left\|\mathbf{Q}_{2}\right\|^{2}+\left\|\mathbf{Q}_{3}\right\|^{2}=2\|\mathbf{L}\|^{2}$,

where \|\| is the Euclidean norm. The material volume fraction, s, can be approximated neglecting the higher order terms as:

$$
\mathrm{s}=\frac{\|\mathbf{L}\|^{2}}{|\mathrm{~V}|}
$$


Taking into account (22-23), (20) can be substituted into (4) giving:

$\Sigma=\frac{1}{|\mathrm{~V}|} \mathbf{S} \cdot \mathbf{N}^{\mathrm{T}}=\frac{1}{|\mathrm{~V}|}\left\{\mathbf{R}_{1}, \mathbf{R}_{2}, \mathbf{R}_{3}, \mathbf{Q}_{1}, \mathbf{Q}_{2}, \mathbf{Q}_{3},\right\}^{\mathrm{T}} \cdot \mathbf{N}^{\mathrm{T}}$

and (21) into (5) as:

$$
\mathrm{W}=\frac{\|\mathbf{N}\|^{2}}{2|\mathrm{~V}| \mathrm{E}_{\mathrm{s}}}
$$

where $\mathbf{S}$ will be named as the modified static matrix.

As written in Section 2.1, a particular engineering constant can be expressed, from (2) or (3), independently of the others, if the corresponding macroload from Table 1 is applied. Then expressions (28-29) can be introduced and the initial expression for the bounding procedure, in terms of normal forces and geometrical parameters, is obtained. The bounding procedure is performed using basic knowledge from linear algebra and the Voigt assumption for the upper bound derivation (uniform local strain), and the bound is finally expressed as a linear function of the material volume fraction. The maximality conditions on possible normal forces are then obtained as conditions that ensure equality with the bound. The specifications in Tab. 1 provide the additional constraints on the possible normal forces that can be developed in an optimal medium. Using the maximality conditions, these additional constraints can be written in terms of microstructure geometrical parameters, as will be seen in Section 3 .

For more details on the Voigt assumption and bound see e.g. Hill (1963). We only remark that, when the local strains are uniform throughout the medium, then they are equal to the macroscopic strain and the global engineering constant corresponding to such a macroload reaches its maximum. Since micro-truss media are characterized by the middle axes of the bars, which (except for the joints) correspond to the "direction" of the local strain, Voigt assumption implies that the local displacements of middle axes of the bars, $\mathbf{u}$, coincide with the linear part of the displacements, i.e. $u_{i}=E_{i j} y_{j}$ (the summation convention is adopted). This requirement states the necessary maximality conditions on possible normal forces, which can be written as:

$$
\mathbf{S}^{\mathrm{T}} \cdot \mathbf{E}=\frac{\mathbf{N}^{\mathrm{T}}}{\mathrm{E}_{\mathrm{s}}}
$$

Maximality conditions (30) are not sufficient, because the requirement of uniform strain does not exclude bars with zero normal force (zero bars). More facts about the relation between optimal micro-frames and the Voigt bound are given in the Appendix.

Obviously, upper bounds determined in the way described in this subsection can be extremely large and unrealistic, because none of the restrictions, e.g., topological connectivity or equilibrium of the joints, were considered. However, if a physical medium saturating the bound can be found, the bound would be proven as optimal. This is actually achieved in all the cases considered in this paper.

\section{Linearized bounds on effective properties}

\subsection{Bulk modulus $\mathrm{K}^{*}$ (for effective isotropy or cubic symmetry)}

If the macroload $\Sigma^{\mathrm{K}}$ (Table 1) is imposed, then starting with (2) and introducing (28-29), (26) and (27), the bulk modulus $\mathrm{K}^{*}$ can be expressed as:

$$
\begin{aligned}
\mathrm{K}^{*} & =\frac{\Sigma_{\mathrm{M}}^{2}}{2 \mathrm{E}_{\mathrm{s}} \mathrm{W}}=\frac{|\mathrm{V}|}{\|\mathbf{N}\|^{2}}\left(\frac{\left(\mathbf{R}_{1}+\mathbf{R}_{2}+\mathbf{R}_{3}\right) \cdot \mathbf{N}^{\mathrm{T}}}{3|\mathrm{~V}|}\right)^{2}= \\
& =\frac{1}{9|\mathrm{~V}|} \cdot \frac{\left(\mathbf{L} \cdot \mathbf{N}^{\mathrm{T}}\right)^{2}}{\|\mathbf{N}\|^{2}} \leq \frac{\mathrm{s}}{9},
\end{aligned}
$$

providing the maximality condition

$$
\mathbf{N} / / \mathbf{L},
$$

(i.e. the local stresses are required to be constant all ever the bars) and the bound $\mathrm{K}_{+}^{*}=\mathrm{s} / 9$. Using (32), additional constraints from Table 1 can be written in terms of geometrical parameters as:

$$
\mathbf{R}_{1} \cdot \mathbf{L}^{\mathrm{T}}=\mathbf{R}_{2} \cdot \mathbf{L}^{\mathrm{T}}=\mathbf{R}_{3} \cdot \mathbf{L}^{\mathrm{T}} \quad \& \quad \mathbf{Q}_{\mathrm{j}} \perp \mathbf{L} \forall \mathrm{j}=1,2,3 .
$$

Equation (30), which should also be implemented, does not in this case bring anything new. It is seen that it corresponds directly to (32), after conditions from Table 1 have been implemented, $\mathbf{N}=\frac{\Sigma}{3 \mathrm{~K}^{*}} \mathbf{L}$. We can check that in this case (30) ensures not only a necessary but also a sufficient maximality condition, because zero bars are excluded as $\mathrm{N}_{\mathrm{i}} \sim \mathrm{A}_{\mathrm{i}} \neq 0 \dot{\forall}$, where " $\sim$ " means proportionality.

The conditions stated in (32-33) are the necessary and sufficient conditions on $\mathrm{K}^{*}$ - optimal media. (32) cannot be expressed only in terms of geometrical parameters, and therefore verification of the $\mathrm{K}^{*}$-optimality of some medium requires the determination of the normal forces in $\mathbf{N}$. The bound is optimal, because several known media saturate it. The simplest $\mathrm{K}^{*}$-optimal medium is a regular cubic lattice (Fig. 5) (see Warren and Kraynik (1988), Dimitrovová (1999)); where it is easy to verify conditions (32-33). The class of periodic $\mathrm{K}^{*}$-optimal media can be extended by the class of media with a random microstructure, where a basic cell of some $\mathrm{K}^{*}$-optimal medium appears in the representative volume

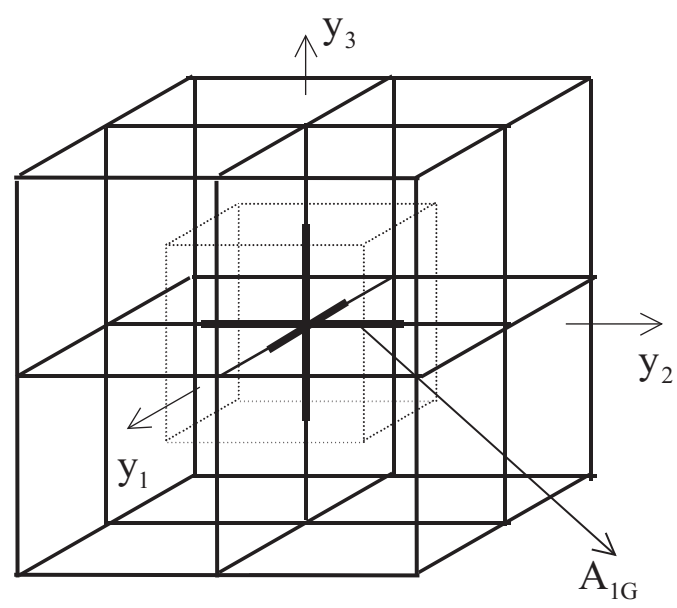

Fig. 5: The regular cubic lattice 
element with all possible rotations with the same probability. Because the bulk modulus is invariant under orientational averaging, the bulk modulus of the new random medium will be the same as for the corresponding periodic medium, Dimitrovová (1999).

\subsection{Shear modulus $\mathrm{G}_{1}^{*}$ (for effective cubic symmetry)}

If macroload $\Sigma^{1 \mathrm{G}}$ (see Table 1) is imposed, then one has:

$$
\begin{gathered}
\mathrm{G}_{1}^{*}=\frac{1}{6|\mathrm{~V}|} \cdot \frac{\left(\mathbf{P}_{3} \cdot \mathbf{N}^{\mathrm{T}}\right)^{2}+\left(\mathbf{P}_{2} \cdot \mathbf{N}^{\mathrm{T}}\right)^{2}+\left(\mathbf{P}_{1} \cdot \mathbf{N}^{\mathrm{T}}\right)^{2}}{\|\mathbf{N}\|^{2}}= \\
\frac{\mathrm{s}}{6} \cdot\left(\frac{\left\|\mathbf{P}_{1}\right\|^{2} \cos ^{2}\left(\mathbf{N}, \mathbf{P}_{1}\right)+\left\|\mathbf{P}_{2}\right\|^{2} \cos ^{2}\left(\mathbf{N}, \mathbf{P}_{2}\right)}{\|\mathbf{L}\|^{2}}+\right. \\
\left.+\frac{\left\|\mathbf{P}_{3}\right\|^{2} \cos ^{2}\left(\mathbf{N}, \mathbf{P}_{3}\right)}{\|\mathbf{L}\|^{2}}\right)= \\
=\frac{\mathrm{s}}{3} \cdot\left(\frac{\left\|\mathbf{P}_{1}\right\|^{2} \cos ^{2}\left(\mathbf{N}, \mathbf{P}_{1}\right)+\left\|\mathbf{P}_{2}\right\|^{2} \cos ^{2}\left(\mathbf{N}, \mathbf{P}_{2}\right)}{\left\|\mathbf{P}_{1}\right\|^{2}+\left\|\mathbf{P}_{2}\right\|^{2}+\left\|\mathbf{P}_{3}\right\|^{2}+\left\|\mathbf{Q}_{1}\right\|^{2}+\left\|\mathbf{Q}_{2}\right\|^{2}+\left\|\mathbf{Q}_{3}\right\|^{2}}+\right. \\
\left.+\frac{\left\|\mathbf{P}_{3}\right\|^{2} \cos ^{2}\left(\mathbf{N}, \mathbf{P}_{3}\right)}{\left\|\mathbf{P}_{1}\right\|^{2}+\left\|\mathbf{P}_{2}\right\|^{2}+\left\|\mathbf{P}_{3}\right\|^{2}+\left\|\mathbf{Q}_{1}\right\|^{2}+\left\|\mathbf{Q}_{2}\right\|^{2}+\left\|\mathbf{Q}_{3}\right\|^{2}}\right)
\end{gathered}
$$

According to Table 1, additional constraints on possible $\mathbf{N}$ are:

$$
\mathbf{N} \mathbb{L} \& \mathbf{N} \mathbf{Q}_{\mathbf{j}} \forall \mathrm{j}=1,2,3 .
$$

If $\mathbf{Q}_{\mathrm{j}}=\mathbf{0} \forall \mathrm{j}=1,2,3$ and $\mathbf{N} / / \mathbf{P}_{\mathrm{j}} \forall \mathrm{j}=1,2,3$, then the maximum in (34) would be $\mathrm{s} / 3$. However none physical medium could fulfill all these conditions, as will be shown in the following. In order to determine the real maximum, it is necessary to realize that any $\Sigma^{1 \mathrm{G}}$ can be written as a linear combination of three basic cases $\Sigma_{22}=-\Sigma_{33}=1, \Sigma_{33}=-\Sigma_{11}=1$ and $\Sigma_{11}=-\Sigma_{22}=1$. In each of them local strains must be uniform according to (30) and the value of the corresponding $G_{1}^{*}$ must be the same, as specified in Table 2 .

Using superposition, the necessary maximality condition from Table 2 reads as:

$\mathbf{N}=\frac{1}{2 G_{1}^{*}}\left(\mu_{1} \mathbf{P}_{1}+\mu_{2} \mathbf{P}_{2}+\mu_{3} \mathbf{P}_{3}\right)=\lambda_{1} \mathbf{P}_{1}+\lambda_{2} \mathbf{P}_{2}+\lambda_{3} \mathbf{P}_{3}$,

where the coefficients $\mu_{\mathrm{i}}$ are expressing the particular basic cases combination, corresponding to the imposed macroload.

Table 2: Basic load cases in $\Sigma^{1 \mathrm{G}}$
Additional constraints from Table 2 must be satisfied simultaneously, giving:

$$
\begin{aligned}
& \mathbf{Q}_{\mathrm{j}} \perp \mathbf{P}_{\mathrm{k}} \forall \mathrm{j}, \mathrm{k}=1,2,3,\left\|\mathbf{R}_{1}\right\|=\left\|\mathbf{R}_{2}\right\|=\left\|\mathbf{R}_{3}\right\|, \\
& \cos \left(\mathbf{R}_{1}, \mathbf{R}_{2}\right)=\cos \left(\mathbf{R}_{2}, \mathbf{R}_{3}\right)=\cos \left(\mathbf{R}_{3}, \mathbf{R}_{1}\right)
\end{aligned}
$$

and

$$
\left\|\mathbf{P}_{1}\right\|=\left\|\mathbf{P}_{2}\right\|=\left\|\mathbf{P}_{3}\right\|=2 \sqrt{\mathrm{G}_{1}^{*}|\mathrm{~V}|} .
$$

(38) could be obtained directly as the condition ensuring the same $G_{1}^{*}$ in all basic cases. If (38) were to be derived first, then using some statements about finite dimensional spaces, condition (36) is the maximality condition for the sum: $\cos ^{2}\left(\mathbf{N}, \mathbf{P}_{1}\right)+\cos ^{2}\left(\mathbf{N}, \mathbf{P}_{2}\right)+\cos ^{2}\left(\mathbf{N}, \mathbf{P}_{3}\right)$. Here it holds:

$$
\cos ^{2}\left(\mathbf{N}, \mathbf{P}_{1}\right)+\cos ^{2}\left(\mathbf{N}, \mathbf{P}_{2}\right)+\cos ^{2}\left(\mathbf{N}, \mathbf{P}_{3}\right)=\frac{3}{2}
$$

Then the bound $\mathrm{G}_{1,+}^{*}=\mathrm{s} / 6$ can be obtained from (34) if $\mathbf{Q}_{\mathrm{j}}=\mathbf{0} \forall \mathrm{j}=1,2,3$. Thus the proof of $\mathrm{G}_{1,+}^{*}=\mathrm{s} / 6$ would be completed if at least one optimal medium can be found, i.e. if there exists a medium in which $\mathbf{Q}_{\mathrm{j}}=\mathbf{0} \forall \mathrm{j}=1,2,3$, expressions (36-38) hold and no zero bars are contained in it.

In order to justify the existence of such a medium, first of all, the spherical angles that will ensure $\mathbf{Q}_{\mathrm{j}}=\mathbf{0} \forall \mathrm{j}=1,2,3$ must be found. This requirement is equivalent to the condition under which

$$
\max \left(\left|\Psi_{1, \mathrm{i}}\right|+\left|\Psi_{2, \mathrm{i}}\right|+\left|\Psi_{3, \mathrm{i}}\right|\right)
$$

is obtained for each i. Solution of problem (40) results in three groups of angles, which predict the bars directions of the bars in an optimal medium, as stated in Table 3. It is therefore convenient to choose a rectangular basic cell with faces perpendicular to the directions of the bars. Due to the equilibrium in the joints, only continuous bars passing through the cell can be present. From Table 3 it follows immediately that $\mathbf{R}_{1} \perp \mathbf{R}_{2} \perp \mathbf{R}_{3}$, but in order to ensure $\left\|\mathbf{R}_{1}\right\|=\left\|\mathbf{R}_{2}\right\|=\left\|\mathbf{R}_{3}\right\|$, the

\begin{tabular}{|c|c|c|c|}
\hline Basic case & 1. & 2. & 3. \\
\hline Macrostress & $\Sigma_{22}=-\Sigma_{33}=1$ & $\Sigma_{33}=-\Sigma_{11}=1$ & $\Sigma_{11}=-\Sigma_{22}=1$ \\
\hline Macrostrain & $\begin{array}{l}\mathrm{E}_{22}=-\mathrm{E}_{33}= \\
=1 /\left(2 \mathrm{G}_{1}^{*} \mathrm{E}_{\mathrm{s}}\right)\end{array}$ & $\begin{array}{l}\mathrm{E}_{33}=-\mathrm{E}_{11}= \\
=1 /\left(2 \mathrm{G}_{1}^{*} \mathrm{E}_{\mathrm{s}}\right)\end{array}$ & $\begin{array}{l}\mathrm{E}_{11}=-\mathrm{E}_{22}= \\
=1 /\left(2 \mathrm{G}_{1}^{*} \mathrm{E}_{\mathrm{s}}\right)\end{array}$ \\
\hline $\begin{array}{l}\text { Maximality condition from } \\
\text { Eq. (30) }\end{array}$ & $\mathbf{N}=\frac{1}{2 \mathrm{G}_{1}^{*}} \mathbf{P}_{1}$ & $\mathbf{N}=\frac{1}{2 \mathrm{G}_{1}^{*}} \mathbf{P}_{2}$ & $\mathbf{N}=\frac{1}{2 \mathrm{G}_{1}^{*}} \mathbf{P}_{3}$ \\
\hline Additional constraints & $\begin{array}{c}\left\|\mathbf{R}_{2}\right\|=\left\|\mathbf{R}_{3}\right\|, \mathbf{R}_{1} \perp \mathbf{P}_{1}, \\
\mathbf{Q}_{\mathrm{j}} \perp \mathbf{P}_{1} \forall \mathbf{j}=1,2,3\end{array}$ & $\begin{array}{c}\left\|\mathbf{R}_{3}\right\|=\left\|\mathbf{R}_{1}\right\|, \mathbf{R}_{2} \perp \mathbf{P}_{2}, \\
\mathbf{Q}_{\mathrm{j}} \perp \mathbf{P}_{2} \forall \mathrm{j}=1,2,3\end{array}$ & $\begin{array}{r}\left\|\mathbf{R}_{1}\right\|=\left\|\mathbf{R}_{2}\right\|, \mathbf{R}_{3} \perp \mathbf{P}_{3}, \\
\mathbf{Q}_{\mathrm{j}} \perp \mathbf{P}_{3} \forall \mathrm{j}=1,2,3\end{array}$ \\
\hline
\end{tabular}
following condition must be satisfied:

$$
\sum_{\text {1.group }} l_{\mathrm{i}} \mathrm{A}_{\mathrm{i}}=\sum_{\text {2.group }} l_{\mathrm{j}} \mathrm{A}_{\mathrm{j}}=\sum_{3 . \text { group }} l_{\mathrm{k}} \mathrm{A}_{\mathrm{k}},
$$

i.e. in each of the three perpendicular directions in the cell, the volume of the bars must be the same. It remains to ensure (36) and impose conditions to eliminate zero bars. Let us take for example one continuous bar from the first group. From (36) it follows that all over the bar $\mathrm{N}_{\mathrm{i}} / \mathrm{A}_{\mathrm{i}}=\lambda_{3}-\lambda_{2}$ holds. Therefore the normal forces between the respective joints must be proportional to the cross sectional areas with the 
Table 3: Characterization of $\mathrm{G}_{1}^{*}$-optimal media

\begin{tabular}{|c|c|c|c|}
\hline Group & 1. & 2. & 3. \\
\hline Spherical angles & $\theta^{1}=\pi / 2, \varphi^{1}=0$ & $\theta^{2}=\pi / 2, \varphi^{2}=\pi / 2$ & $\theta^{3}=0$ \\
\hline Values of $\Psi_{1}, \Psi_{2}, \Psi_{3}$ & $0,-1,1$ & $1,0,-1$ & $-1,1,0$ \\
\hline Values of $\Omega_{1}, \Omega_{2}, \Omega_{3}$ & $1,0,0$ & $0,1,0$ & $0,0,1$ \\
\hline
\end{tabular}

same coefficient of proportionality in each group. Due to the equilibrium in the joints, the normal forces must be the same within each continuous bar, which implies that the cross sectional areas are also constant within the continuous bar, as well.

Let us now summarize the results. $G_{1,+}^{*}=s / 6$ and all $G_{1}^{*}$ -optimal media can be fully geometrically specified in the following way:

$\mathrm{G}_{1}^{*}$-optimal media are continuous lattices for which:

- a rectangular basic cell (with dimensions $\mathrm{L}_{\mathrm{i}}$ in $\mathrm{y}_{\mathrm{i}}$-directions, $\mathrm{i}=1,2,3$ ) can be found, consisting only of continuous orthogonal bars in $\mathrm{y}_{\mathrm{i}}$-directions,

- each bar has a constant cross sectional area within the basic cell and the condition $\mathrm{L}_{1} \sum_{\mathrm{i}=1}^{\mathrm{n}_{1}} \mathrm{~A}_{\mathrm{i}}=\mathrm{L}_{2} \sum_{\mathrm{j}=1}^{\mathrm{n}_{2}} \mathrm{~A}_{\mathrm{j}}=\mathrm{L}_{3} \sum_{\mathrm{k}=1}^{\mathrm{n}_{3}} \mathrm{~A}_{\mathrm{k}}$ is satisfied $\left(n_{i}\right.$ is the number of bars in the $y_{i}$-direction, $i=1$, 2, 3).

The group of media specified above is the only group of $\mathrm{G}_{1}^{*}$-optimal media. They are in fact a $3 \mathrm{D}$ extension of UPL (the uniform perpendicular lattices) introduced in Dimitrovová and Faria (1999). The simplest example from this group is the regular cubic lattice (Fig. 5). The value of its $G_{1}^{*}$ (not the proof of maximality) can be obtained directly from $G_{1}^{*}$ of its 2D analog: the regular square lattice. If we denote by $\mathrm{s}_{2 \mathrm{D}}$ and $\mathrm{S}_{3 \mathrm{D}}$ the material volume fractions of $2 \mathrm{D}$ and $3 \mathrm{D}$ regular lattices, respectively, it holds $\mathrm{s}_{2 \mathrm{D}}=2 \mathrm{~s}_{3 \mathrm{D}} / 3$, and consequently

$$
\mathrm{G}_{1}^{*}=\frac{1}{4} \mathrm{~s}_{2 \mathrm{D}}=\frac{1}{6} \mathrm{~s}_{3 \mathrm{D}} .
$$

\subsection{Shear modulus $\mathrm{G}_{2}^{*}$ (for effective cubic symmetry)}

First of all, we point out that in 3D there exists no such rotation of the global coordinates that would interchange the positions of $G_{1}^{*}$ and $G_{2}^{*}$ in $\mathbf{C}^{*}$, as it does in $2 \mathrm{D}$ (see Dimitrovová and Faria (1999)). Thus $\mathrm{G}_{2}^{*}$-optimal media cannot be derived from $\mathrm{G}_{1}^{*}$-optimal media. For macroload $\Sigma^{2 \mathrm{G}}$ we can obtain:

$$
\begin{gathered}
\mathrm{G}_{2}^{*}=\frac{1}{6|\mathrm{~V}|} \cdot \frac{\left(\mathbf{Q}_{1} \cdot \mathbf{N}^{\mathrm{T}}\right)^{2}+\left(\mathbf{Q}_{2} \cdot \mathbf{N}^{\mathrm{T}}\right)^{2}+\left(\mathbf{Q}_{3} \cdot \mathbf{N}^{\mathrm{T}}\right)^{2}}{\|\mathbf{N}\|^{2}}= \\
=\frac{\mathrm{s}}{3} \cdot\left(\frac{\left\|\mathbf{Q}_{1}\right\|^{2} \cos ^{2}\left(\mathbf{N}, \mathbf{Q}_{1}\right)+\left\|\mathbf{Q}_{2}\right\|^{2} \cos ^{2}\left(\mathbf{N}, \mathbf{Q}_{2}\right)}{\left\|\mathbf{P}_{1}\right\|^{2}+\left\|\mathbf{P}_{2}\right\|^{2}+\left\|\mathbf{P}_{3}\right\|^{2}+\left\|\mathbf{Q}_{1}\right\|^{2}+\left\|\mathbf{Q}_{2}\right\|^{2}+\left\|\mathbf{Q}_{3}\right\|^{2}}+\right. \\
\left.+\frac{\left\|\mathbf{Q}_{3}\right\|^{2} \cos ^{2}\left(\mathbf{N}, \mathbf{Q}_{3}\right)}{\left\|\mathbf{P}_{1}\right\|^{2}+\left\|\mathbf{P}_{2}\right\|^{2}+\left\|\mathbf{P}_{3}\right\|^{2}+\left\|\mathbf{Q}_{1}\right\|^{2}+\left\|\mathbf{Q}_{2}\right\|^{2}+\left\|\mathbf{Q}_{3}\right\|^{2}}\right) .
\end{gathered}
$$

Additional constraints on possible $\mathbf{N}$ are:

$$
\mathbf{N} \perp \mathbf{R}_{\mathrm{j}} \forall \mathrm{j}=1,2,3 .
$$

The obvious maximum s/3 cannot be achieved by any medium, similarly as in Section 3.2. Also, combining the 2D results (unlike to (42)) would lead to a wrong conclusion, as can be demonstrated: let only $\Sigma_{23} \neq 0$ in $\Sigma^{2 \mathrm{G}}$, then an optimal medium should have bars in the directions of the unit square diagonals in $(2,3)$-planes, according to Dimitrovová and Faria (1999). By analogy, the other load cases $\Sigma_{12} \neq 0$ and $\Sigma_{13} \neq 0$

\begin{tabular}{|c|c|c|c|}
\hline Basic case & 1. & 2. & 3. \\
\hline Macrostress & $\Sigma_{23}=1$ & $\Sigma_{13}=1$ & $\Sigma_{12}=1$ \\
\hline Macrostrain & $\Sigma_{23}=1 /\left(2 \mathrm{G}_{2}^{*} \mathrm{E}_{\mathrm{s}}\right)$ & $\Sigma_{13}=1 /\left(2 \mathrm{G}_{2}^{*} \mathrm{E}_{\mathrm{s}}\right)$ & $\Sigma_{12}=1 /\left(2 \mathrm{G}_{2}^{*} \mathrm{E}_{\mathrm{s}}\right)$ \\
\hline $\begin{array}{l}\text { Maximality condition from } \\
\text { Eq. (30) }\end{array}$ & $\mathbf{N}=\frac{1}{{ }^{2} \mathrm{G}^{*}} \mathbf{Q}_{1}$ & $\mathbf{N}=\frac{1}{{ }^{2} \mathrm{G}^{*}} \mathbf{Q}_{2}$ & $\mathbf{N}=\frac{1}{{ }^{2} \mathrm{G}^{*}} \mathbf{Q}_{3}$ \\
\hline Additional constraints & $\begin{array}{l}{ }^{1} \mathbf{Q} \perp{ }^{2} \mathbf{Q} \&{ }^{1} \mathbf{Q} \perp{ }^{3} \mathbf{Q} \\
{ }^{1} \mathbf{Q} \perp{ }^{\mathrm{i}} \mathbf{R} \forall \dot{ } \\
\left\|{ }^{1} \mathbf{Q}\right\|^{2}={ }^{2} \mathrm{G}^{*}|\mathrm{~V}|\end{array}$ & $\begin{array}{l}{ }^{2} \mathbf{Q} \perp{ }^{1} \mathbf{Q} \&{ }^{2} \mathbf{Q} \perp{ }^{3} \mathbf{Q} \\
{ }^{2} \mathbf{Q} \perp{ }^{\mathrm{i}} \mathbf{R} \forall \\
\left\|{ }^{2} \mathbf{Q}\right\|^{2}={ }^{2} \mathrm{G}{ }^{*}|\mathrm{~V}|\end{array}$ & $\begin{array}{l}{ }^{3} \mathbf{Q} \perp{ }^{1} \mathbf{Q} \&{ }^{3} \mathbf{Q} \perp{ }^{2} \mathbf{Q} \\
{ }^{3} \mathbf{Q} \perp{ }^{\mathrm{i}} \mathbf{R} \forall \\
\left\|{ }^{3} \mathbf{Q}\right\|^{2}={ }^{2} \mathrm{G}^{*}|\mathrm{~V}|\end{array}$ \\
\hline
\end{tabular}
imply directions of the bars in $(1,2)$ and $(1,3)$-planes, respectively. The $2 \mathrm{D}$ result $\mathrm{G}_{2,+}^{*}=\mathrm{s}_{2 \mathrm{D}} / 4$ and the fact that $\mathrm{s}_{2 \mathrm{D}}=\mathrm{s}_{3 \mathrm{D}} / 3$ thus yield $\mathrm{G}_{2}^{*}=\mathrm{s}_{3 \mathrm{D}} / 12$, because the directions of

Table 4: Basic load cases in $\Sigma^{2 \mathrm{G}}$ 
Table 5: Characterization of $\mathrm{G}_{2}^{*}$-optimal media

\begin{tabular}{|c|c|c|c|c|}
\hline Group & 1. & 2. & 3. & 4. \\
\hline \multirow{2}{*}{ Spherical angles } & $\cos \varphi^{1}=\frac{1}{\sqrt{2}} ;$ & $\cos \varphi^{2}=\frac{1}{\sqrt{2}} ;$ & $\cos \varphi^{3}=-\frac{1}{\sqrt{2}} ;$ & $\cos \varphi^{4}=-\frac{1}{\sqrt{2}} ;$ \\
& $\sin \varphi^{1}=\frac{1}{\sqrt{2}} ;$ & $\sin \varphi^{2}=\frac{1}{\sqrt{2}} ;$ & $\sin \varphi^{3}=\frac{1}{\sqrt{2}} ;$ & $\sin \varphi^{4}=\frac{1}{\sqrt{2}} ;$ \\
& $\cos \theta^{1}=\frac{1}{\sqrt{3}} ;$ & $\cos \theta^{2}=-\frac{1}{\sqrt{3}} ;$ & $\cos \theta^{3}=\frac{1}{\sqrt{3}} ;$ & $\cos \theta^{4}=-\frac{1}{\sqrt{3}} ;$ \\
\hline Values of $\Phi_{1}, \Phi_{2}, \Phi_{3}$ & $\sin \theta^{1}=\frac{\sqrt{2}}{\sqrt{3}}$ & $\sin \theta^{2}=\frac{\sqrt{2}}{\sqrt{3}}$ & $\sin \theta^{3}=\frac{\sqrt{2}}{\sqrt{3}}$ & $\sin \theta^{4}=\frac{\sqrt{2}}{\sqrt{3}}$ \\
\hline Values of $\Omega_{1}, \Omega_{2}, \Omega_{3}$ & $1 / 3,1 / 3,1 / 3$ & $-1 / 3,-1 / 3,1 / 3$ & $1 / 3,-1 / 3,-1 / 3$ & $-1 / 3,1 / 3,-1 / 3$ \\
\hline
\end{tabular}

the bars stated previously do not coincide. However, it will be proven that $\mathrm{G}_{2,+}^{*}=\mathrm{s} / 9$.

An arbitrary $\Sigma^{2 \mathrm{G}}$ can be expressed as a linear combination of three basic cases (Table 4). Using superposition, the necessary maximality condition reads as:

$\mathbf{N}=\frac{1}{G_{2}^{*}}\left(\mu_{1} \mathbf{Q}_{1}+\mu_{2} \mathbf{Q}_{2}+\mu_{3} \mathbf{Q}_{3}\right)=\lambda_{1} \mathbf{Q}_{1}+\lambda_{2} \mathbf{Q}_{2}+\lambda_{3} \mathbf{Q}_{3}$,

where the coefficients $\mu_{\mathrm{i}}$ express a combination of the specific basic cases, corresponding to the imposed macroload. Additional constraints are:

$$
\mathbf{Q}_{\mathrm{j}} \perp \mathbf{R}_{\mathrm{k}} \forall \mathrm{j}, \mathrm{k}=1,2,3 \& \mathbf{Q}_{1} \perp \mathbf{Q}_{2} \perp \mathbf{Q}_{3}
$$

and

$$
\left\|\mathbf{Q}_{1}\right\|=\left\|\mathbf{Q}_{2}\right\|=\left\|\mathbf{Q}_{3}\right\|=\sqrt{\mathrm{G}_{2}^{*}|\mathrm{~V}|}
$$

If (47) were to be derived first, then using some statements about finite dimensional spaces, (45) is the maximality condition for the sum of cosines from (43), similarly as in Section 3.2. Now, due to the orthogonality of $\mathbf{Q}_{\mathrm{j}}$, the sum of the cosines is equal to 1 , therefore $\mathrm{G}_{2,+}^{*}=\mathrm{s} / 9$, if at least one optimal medium exists, i.e. if there can be found a medium in which $\mathbf{P}_{\mathrm{j}}=\mathbf{0} \forall \mathrm{j}=1,2,3$, (45-47) hold and no zero bars are contained in it. The requirement $\mathbf{P}_{\mathrm{j}}=\mathbf{0} \forall \mathrm{j}=1,2,3$ is equivalent to the condition under which

$$
\max \left(\left|\Phi_{1, \mathrm{i}}\right|+\left|\Phi_{2, \mathrm{i}}\right|+\left|\Phi_{3, \mathrm{i}}\right|\right)
$$

is obtained for each i. Solutions of (48) yield four groups of angles, as specified in Table 5 , corresponding to the main diagonals of the unit cube. It is not convenient to choose a basic cell with eight faces (perpendicular to the directions of the bars), because a regular octahedron does not fill the space. It is better to assume a rectangular cell according to Fig. 6. Conditions $\mathbf{Q}_{\mathrm{j}} \perp \mathbf{R}_{\mathrm{k}} \forall \mathrm{j}, \mathrm{k}=1,2,3$ imply, again, the same volume constraint of the bars within each group:

$$
\sum_{\text {1.group }} l_{\mathrm{i}} \mathrm{A}_{\mathrm{i}}=\sum_{\text {2.group }} l_{\mathrm{j}} \mathrm{A}_{\mathrm{j}}=\sum_{3 \text {.group }} l_{\mathrm{k}} \mathrm{A}_{\mathrm{k}}=\sum_{\text {4.group }} l_{\mathrm{r}} \mathrm{A}_{\mathrm{r}} \text {, }
$$

which, in a sequel, guarantees the mutual perpendicularity of $\mathbf{Q}_{\mathrm{j}}, \mathrm{j}=1,2,3$, while (47) is satisfied directly.

It remains to ensure (45) and impose conditions to eliminate zero bars. Let us take one bar from the first group. From (45) it directly follows that in each part between the joints of

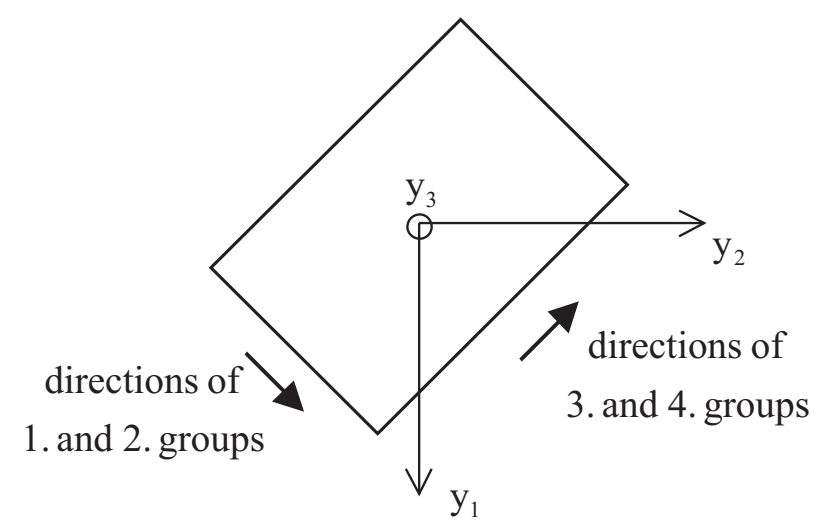

Fig. 6: Rectangular cell for $\mathrm{G}_{2}^{*}$-optimal media, top view

this bar $\mathrm{N}_{\mathrm{i}} / \mathrm{A}_{\mathrm{i}}=\left(-\lambda_{1}+\lambda_{2}-\lambda_{3}\right) / 3$ holds. Thus the normal forces must be proportional to the cross sectional areas with the same coefficient of proportionality in each group, in other words, in the bars of each group the local stresses must be the same: $\sigma_{1}, \sigma_{2}, \sigma_{3}, \sigma_{4}$, respectively. Because four possible directions of the bars exist, it cannot be directly concluded that due to the equilibrium in the joints only continuous bars are included in the cell. However, this statement can be justified in the following way. Obviously

$$
\begin{aligned}
& \sigma_{1}=\left(-\lambda_{1}+\lambda_{2}-\lambda_{3}\right) / 3, \\
& \sigma_{2}=\left(\lambda_{1}-\lambda_{2}-\lambda_{3}\right) / 3, \\
& \sigma_{3}=\left(-\lambda_{1}-\lambda_{2}+\lambda_{3}\right) / 3 \text { and } \\
& \sigma_{4}=\left(\lambda_{1}+\lambda_{2}+\lambda_{3}\right) / 3
\end{aligned}
$$

hold. Let us take a joint and suppose that a member from each group is presented there as continuous. Contributions to the cell coordinate directions are given in Table 6. Consequently, the equilibrium in the joint reads as:

$$
\begin{aligned}
& \sigma_{1}\left(\mathrm{~A}_{1, \mathrm{~m}}-\mathrm{A}_{1, \mathrm{~m}-1}\right)+\sigma_{2}\left(\mathrm{~A}_{2, \mathrm{n}}-\mathrm{A}_{2, \mathrm{n}-1}\right)- \\
& -\sigma_{3}\left(\mathrm{~A}_{3, \mathrm{r}}-\mathrm{A}_{3, \mathrm{r}-1}\right)-\sigma_{4}\left(\mathrm{~A}_{4, \mathrm{~s}}-\mathrm{A}_{4, \mathrm{~s}-1}\right)=0, \\
& \sigma_{1}\left(\mathrm{~A}_{1, \mathrm{~m}}-\mathrm{A}_{1, \mathrm{~m}-1}\right)+\sigma_{2}\left(\mathrm{~A}_{2, \mathrm{n}}-\mathrm{A}_{2, \mathrm{n}-1}\right)+ \\
& +\sigma_{3}\left(\mathrm{~A}_{3, \mathrm{r}}-\mathrm{A}_{3, \mathrm{r}-1}\right)+\sigma_{4}\left(\mathrm{~A}_{4, \mathrm{~s}}-\mathrm{A}_{4, \mathrm{~s}-1}\right)=0, \\
& \sigma_{1}\left(\mathrm{~A}_{1, \mathrm{~m}}-\mathrm{A}_{1, \mathrm{~m}-1}\right)-\sigma_{2}\left(\mathrm{~A}_{2, \mathrm{n}}-\mathrm{A}_{2, \mathrm{n}-1}\right)+ \\
& +\sigma_{3}\left(\mathrm{~A}_{3, \mathrm{r}}-\mathrm{A}_{3, \mathrm{r}-1}\right)-\sigma_{4}\left(\mathrm{~A}_{4, \mathrm{~s}}-\mathrm{A}_{4, \mathrm{~s}-1}\right)=0,
\end{aligned}
$$


Table 6: Contributions of the local stresses to the coordinate directions

\begin{tabular}{|c|c|c|c|c|}
\hline & $\sigma_{1}$ & $\sigma_{2}$ & $\sigma_{3}$ & $\sigma_{4}$ \\
\hline$y_{1}$ & $1 / \sqrt{3}$ & $1 / \sqrt{3}$ & $-1 / \sqrt{3}$ & $-1 / \sqrt{3}$ \\
\hline$y_{2}$ & $1 / \sqrt{3}$ & $1 / \sqrt{3}$ & $1 / \sqrt{3}$ & $1 / \sqrt{3}$ \\
\hline$y_{3}$ & $1 / \sqrt{3}$ & $-1 / \sqrt{3}$ & $1 / \sqrt{3}$ & $-1 / \sqrt{3}$ \\
\hline
\end{tabular}

where the first subscript at the cross sectional areas denotes the group and the second one expresses the order number within the group. (51) must be satisfied for any $\sigma_{i}, i=1,2,3,4$, consequently the cross sectional areas must be either the same (resulting in a continuous bar with a constant cross sectional area) or zero (the group is not contained in the joint), which completes the justification.

In summary, $\mathrm{G}_{2,+}^{*}=\mathrm{s} / 9$ and all $\mathrm{G}_{2}^{*}$-optimal media can be fully geometrically specified in the following way:

$\mathrm{G}_{2}^{*}$-optimal media are continuous lattices for which:

- a rectangular basic cell, according to Fig. 6, can be found, where only continuous bars in the four directions specified by Table 5 are present,

- each continuous bar has a constant cross sectional area and (49) holds.

The group of media described above is the only group of $\mathrm{G}_{2}^{*}$-optimal media. The name for such media was introduced in Dimitrovová and Faria (1999) as UDL (Uniform Diagonal Lattices). The simplest example from this group is the regular cube-diagonal lattice in Fig. 7.

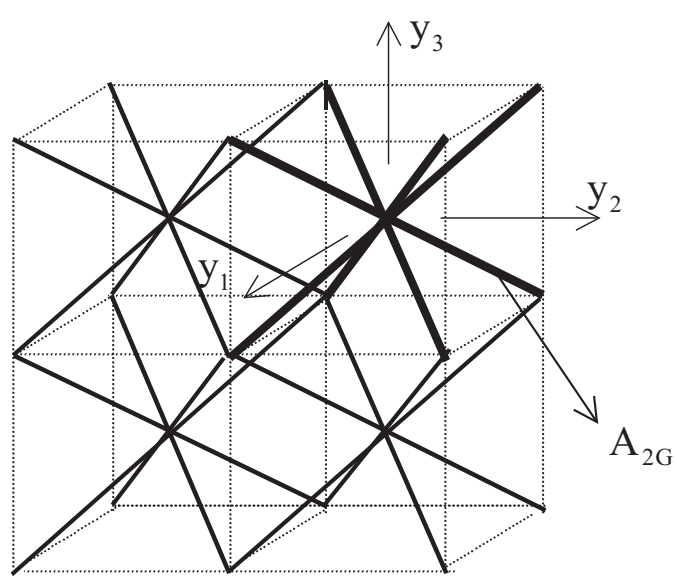

Fig. 7: The regular cube-diagonal lattice

\subsection{Shear modulus $\mathbf{G}^{*}$ (for effective isotropy)}

In this case, the conclusions from the two previous sections can be exploited. Let us assume that we already have a $\mathrm{G}^{*}$-optimal medium. Macroloads $\Sigma^{1 \mathrm{G}}$ and $\Sigma^{2 \mathrm{G}}$ can be imposed separately on it and the same bounding procedure as in Sections 3.3-4 can be performed. It is only necessary to prevent a geometrical specification which would enter in contradiction with the possibility of effective isotropy of the medium. Thus:

$$
\mathrm{G}^{*} \leq \frac{\mathrm{s}}{4} \cdot \frac{\|\mathbf{P}\|^{2}}{\|\mathbf{L}\|^{2}} \text { and } \mathrm{G}^{*} \leq \frac{\mathrm{s}}{6} \cdot \frac{\|\mathbf{Q}\|^{2}}{\|\mathbf{L}\|^{2}},
$$

where the subscripts in $\mathbf{P}$ and $\mathbf{Q}$ are omitted for the sake of simplicity. Since the maximum in both relations of (52) must be the same, $\|\mathbf{P}\|^{2}=2\|\mathbf{Q}\|^{2} / 3$ and taking into account the last expression of (26), $\mathrm{G}^{*} \leq \mathrm{s} / 15$ can finally be obtained. Therefore $G_{+}^{*}=s / 15$, if at least one optimal medium exists. The necessary maximality and additional constraints can be expressed by analogy with Sections 3.2-3 as:

$$
\mathbf{N}=\lambda_{\mathrm{j}} \mathbf{P}_{\mathrm{j}}+\mu_{\mathrm{k}} \mathbf{Q}_{\mathrm{k}}, \mathrm{j}, \mathrm{k}=1,2,3
$$

and

$\left\|\mathbf{R}_{1}\right\|=\left\|\mathbf{R}_{2}\right\|=\left\|\mathbf{R}_{3}\right\|$,

$\cos \left(\mathbf{R}_{1}, \mathbf{R}_{2}\right)=\cos \left(\mathbf{R}_{2}, \mathbf{R}_{3}\right)=\cos \left(\mathbf{R}_{3}, \mathbf{R}_{1}\right) ;$

$\mathbf{Q}_{\mathrm{j}} \perp \mathbf{R}_{\mathrm{k}} \forall \mathrm{j}, \mathrm{k}=1,2,3$;

$\mathbf{Q}_{1} \perp \mathbf{Q}_{2} \perp \mathbf{Q}_{3} ;\left\|\mathbf{Q}_{1}\right\|=\left\|\mathbf{Q}_{2}\right\|=\left\|\mathbf{Q}_{3}\right\|$.

Unfortunately, no full geometrical characterization of $\mathrm{G}^{*}$-optimal media is possible. The existence of at least one optimal medium can be proven by superposition of the results, namely by combining of the cells of the simplest $\mathrm{G}_{1}^{*}$ - and $\mathrm{G}_{2}^{*}$-optimal media, see Dimitrovová and Faria (1999) for the conditions under which such a superposition can be performed. Let us denote the material volume fractions of the

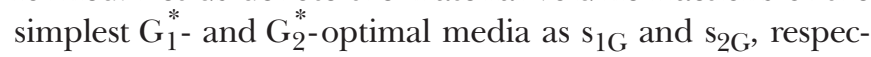
tively. It can be written:

$$
\mathrm{G}^{*}=\frac{\mathrm{s}_{1 \mathrm{G}}}{6}=\frac{\mathrm{s}_{2 \mathrm{G}}}{9},
$$

yielding $s_{1 G}=2 s_{2 G} / 3$ and consequently $\left(s=s_{1 G}+s_{2 G}\right.$ because the bars of the original media do not coincide) $\mathrm{G}^{*}=\mathrm{s} / 15$ for the combined medium. As a consequence, the relation between the cross sectional areas can be derived as $A_{1 G}=8 \sqrt{3} A_{2 G} / 9$, where, $A_{1 G}$ and $A_{2 G}$ stand for the cross sections of the original $G_{1}^{*}$ and $G_{2}^{*}$-optimal media, respectively (Figs. 5 and 7). It can be verified that also in this case the bending effect can be superposed directly, as in the 2D analog, as shown in Dimitrovová and Faria (1999).

\section{Concluding remarks}

It was proven that $\mathrm{G}_{1}^{*}$ - and $\mathrm{G}_{2}^{*}$-optimal media can be geometrically fully specified. They are UPL and UDL, respectively. Neither for $\mathrm{K}^{*}$-optimal nor for $\mathrm{G}^{*}$-optimal media can a full geometrical specification of their microstructure be given. 
It is easy to verify that $G_{1}^{*}$ - and $K^{*}$-optimal media, assumed either as micro-trusses or as micro-frames, respond only axially, while in $\mathrm{G}_{2}^{*}$ and $\mathrm{G}^{*}$-optimal micro-frames, a bending response is always presented. The bending contribution is different for different $G_{2}^{*}$ and $G^{*}$-optimal media, therefore the non-linear part is rather difficult to define. However, it can be stated that for low-density media this non-linear part is not important. The bending contribution can be increased by putting more material close to the joints, because the bending moment distribution is antisymmetric within each beam (Section 2.2). However, this change would decrease the axial contribution (17-18), and the corresponding linearized bound would decrease. Then the medium would no longer be optimal according to the definition from Section 2.2.

It is useful to remark that the directions of the bars in optimal micro-trusses should be related to the principal directions of the applied macroload according to the theory of Michell trusses. This is directly related to the impossibility of full geometrical characterization of $\mathrm{K}^{*}$ - and $\mathrm{G}^{*}$-optimal media. For $\Sigma^{\mathrm{K}}$ each direction is a principal direction. $\Sigma^{\mathrm{G}}$ can be determined by five non-zero and independent parameters, and therefore each direction can also be assumed as a principal one. The directions of the bars are precisely specified in $\mathrm{G}_{1^{-}}^{*}$ and $G_{2}^{*}$-optimal media. In the former case they coincide with the principal directions of the macroload, which are in this case unique for any $\Sigma^{1 \mathrm{G}}$, but in the latter case the directions of the bars can hardly be determined in such a way.

Another remark yields from a comparison of the additional constraints (geometrical requirements) for $\mathrm{K}^{*}$ - and $\mathrm{G}^{*}$-optimal media, (33) and (54), respectively. It can be shown, with the help of (24) and the second relation in (26), that the group of media satisfying (54) forms a subgroup of microstructures for which (33) holds. Therefore each $\mathrm{K}^{*}$-optimal medium already fulfills additional constraints for $\mathrm{G}^{*}$-optimal media, so that it is hard to find a $G^{*}$-optimal medium which is not $\mathrm{K}^{*}$-optimal.

It is straightforward to derive bounds for Young's modulus for media with effective isotropy and cubic symmetry, respectively, in the forms of

$$
\mathrm{E}_{\mathrm{is},+}^{*}=\frac{4 \mathrm{~K}_{+}^{*} \mathrm{G}_{+}^{*}}{\mathrm{~K}_{+}^{*}+\mathrm{G}_{+}^{*}}=\frac{4 \frac{\mathrm{s}}{9} \cdot \frac{\mathrm{s}}{15}}{\frac{\mathrm{s}}{9}+\frac{\mathrm{s}}{15}}=\frac{\mathrm{s}}{6},
$$

$$
\mathrm{E}_{\mathrm{cs},+}^{*}=\frac{4 \mathrm{~K}_{+}^{*} \mathrm{G}_{1,+}^{*}}{\mathrm{~K}_{+}^{*}+\mathrm{G}_{1,+}^{*}}=\frac{4 \frac{\mathrm{s}}{9} \cdot \frac{\mathrm{s}}{6}}{\frac{\mathrm{s}}{9}+\frac{\mathrm{s}}{6}}=\frac{4}{15} \mathrm{~s},
$$

but no conclusions can be reached on the upper bounds on effective Poisson's ratios. It is only easy to verify that UPL have zero effective Poisson's ratio. $\mathrm{K}^{*}-\& \mathrm{G}^{*}$-optimal micro-trusses have an effective Poisson's ratio equal to $1 / 4$, as shown by Bakhvalov and Panasenko (1989).

In optimal micro-trusses it is interesting to see what the other elastic properties are. A summary is given in Table 7 . Furthermore, in Table 8 the bounds for open-cell foams proven in this paper are compared with the composite ones. The composite bounds for effectively isotropic media are taken from Hashin and Shtrikman (1963) and Hashin (1970, 1983 ) and for media with effective cubic symmetry they are taken from Avellaneda (1987). They are specified to one void phase and linearized with respect to the material volume fraction. It can be seen that the solid phase Poisson's ratio $v_{\mathrm{s}}$ naturally appears in the linearized composite bounds (unlike the 2D case shown in Dimitrovová and Faria (1999)). This is because shell or plate parts must be included in optimal 3D media. $\beta$ and $\eta$ stand for coefficients of the bending contribution.

Finally, let us make some remarks about the simplified assumptions adopted for the strain energy contribution. It is known that assuming the micro-frame medium with theoretical lengths makes the medium softer than it really is. It is thus better to use active lengths of the beam and include the deformation of the joints. Moreover, the strain energy density corresponding to the shear forces can be included in W. Obviously, such improvements do not change the linearized bounds since they do not influence expressions for the axial response of the media. If, e.g., the strain energy density corresponding to the shear forces were to be included, parameters $\beta$ and $\eta$ from Tab. 8 would decrease. In this case the solid phase Poisson's ratio would appear in the final result.

\section{Appendix}

Admitting a more general solid material behavior it can be shown that open-cell foam bounds coincide with the Voigt bound. For the sake of simplicity let us assume a 2D medium, the regular lattice, which is $\mathrm{k}^{*}-\& \mathrm{G}_{1}^{*}$-optimal (Dimitrovová and Faria (1999)). $\mathrm{k}^{*}$ stands for 2D bulk modulus and has the

\begin{tabular}{|c|c|c|c|c|c|}
\hline \multirow[t]{2}{*}{ Macro-load } & \multirow[t]{2}{*}{ Optimal micro-trusses } & \multicolumn{4}{|c|}{ Other elastic properties } \\
\hline & & $\mathrm{K}^{*}$ & ${ }^{1} G^{*}$ & ${ }^{2} G^{*}$ & $\mathrm{G}^{*}$ \\
\hline$\Sigma^{\mathrm{K}}$ & $\begin{array}{l}\text { UPL, UDL, other media, which cannot } \\
\text { be fully geometrically specified }\end{array}$ & $\mathrm{K}_{+}^{*}$ & $\begin{array}{l}\text { not uniquely } \\
\text { defined }\end{array}$ & $\begin{array}{l}\text { not uniquely } \\
\text { defined }\end{array}$ & $\begin{array}{l}\text { not uniquely } \\
\text { defined }\end{array}$ \\
\hline$\Sigma^{1 \mathrm{G}}$ & only UPL & $\mathrm{K}_{+}^{*}$ & ${ }^{1} G_{+}^{*}$ & 0 & - \\
\hline$\Sigma^{2 \mathrm{G}}$ & only UDL & $\mathrm{K}_{+}^{*}$ & 0 & ${ }^{2} \mathrm{G}_{+}^{*}$ & - \\
\hline$\Sigma^{\mathrm{G}}$ & $\begin{array}{l}\text { media, which cannot be fully } \\
\text { geometrically specified, but neither } \\
\text { UPL nor UDL }\end{array}$ & $\begin{array}{l}\text { not } \\
\text { uniquely } \\
\text { defined }\end{array}$ & $\mathrm{G}_{+}^{*}$ & $\mathrm{G}_{+}^{*}$ & $\mathrm{G}_{+}^{*}$ \\
\hline
\end{tabular}

Table 7: Other elastic properties in optimal micro-trusses 
Table 8: : Composite bounds, bounds for open-cell foams and characterization of the optimal media

\begin{tabular}{|c|c|c|c|c|}
\hline \multicolumn{5}{|c|}{ Bounds comparison } \\
\hline \multirow{3}{*}{ Composite bounds } & \multicolumn{2}{|c|}{$\mathrm{K}_{+}^{*}=\frac{2 \mathrm{~s}}{3\left(3\left(1-v_{\mathrm{s}}\right)-\mathrm{s}\left(1+\mathrm{v}_{\mathrm{s}}\right)\right)}$} & \multicolumn{2}{|c|}{$\mathrm{G}_{+}^{*}=\frac{\mathrm{s}\left(7-5 v_{\mathrm{s}}\right)}{2\left(1+v_{\mathrm{s}}\right)\left(15\left(1-v_{\mathrm{s}}\right)-2 \mathrm{~s}\left(4-5 v_{\mathrm{s}}\right)\right)}$} \\
\hline & \multicolumn{2}{|c|}{${ }^{1} G_{+}^{*}=\frac{s\left(2-v_{s}\right)}{2\left(1+v_{s}\right)} \cdot \frac{1}{\left(3\left(1-v_{s}\right)-s\left(1-2 v_{s}\right)\right)}$} & \multicolumn{2}{|c|}{${ }^{2} G_{+}^{*}=\frac{s\left(5-3 v_{s}\right)}{2\left(1+v_{s}\right)\left(9\left(1-v_{s}\right)-2 s\left(2-3 v_{s}\right)\right)}$} \\
\hline & $\mathrm{K}_{+}^{*}$ & ${ }^{1} G_{+}^{*}$ & ${ }^{2} \mathrm{G}_{+}^{*}$ & $\mathrm{G}_{+}^{*}$ \\
\hline Linearized form & $\frac{2}{9\left(1-v_{s}\right)} s$ & $\frac{2-v_{s}}{6\left(1-v_{s}^{2}\right)} s$ & $\frac{5-3 v_{\mathrm{s}}}{18\left(1-v_{\mathrm{s}}^{2}\right)} \mathrm{s}$ & $\frac{7-5 v_{s}}{30\left(1-v_{s}^{2}\right)} s$ \\
\hline $\begin{array}{l}\text { Previous form with } \\
\qquad v_{\mathrm{s}}=0\end{array}$ & $\frac{2 \mathrm{~s}}{9}$ & $\frac{\mathrm{s}}{3}$ & $\frac{5 \mathrm{~s}}{18}$ & $\frac{7 \mathrm{~s}}{30}$ \\
\hline $\begin{array}{l}\text { Bound for open-cell } \\
\text { foams from this article }\end{array}$ & $\frac{s}{9}$ & $\frac{s}{6}$ & $\frac{s}{9}+\beta s^{2}$ & $\frac{s}{15}+\eta s^{2}$ \\
\hline Optimal media are ... & $\begin{array}{c}\text { determined by } \\
\text { necessary and sufficient } \\
\text { conditions }\end{array}$ & $\begin{array}{l}\text { fully geometrically } \\
\text { specified }\end{array}$ & $\begin{array}{l}\text { fully geometrically } \\
\text { specified }\end{array}$ & $\begin{array}{c}\text { determined by } \\
\text { necessary and sufficient } \\
\text { conditions }\end{array}$ \\
\hline
\end{tabular}

same meaning as before. Voigt bounds $\mathrm{k}_{\mathrm{V}}^{*}$ and $\mathrm{G}_{1, \mathrm{~V}}^{*}$ for one void phase are (see Hill (1963)):

$$
\mathrm{k}_{\mathrm{V}}^{*}=\frac{\mathrm{s}}{2\left(1-v_{\mathrm{s}}\right)}, \quad \mathrm{G}_{1, \mathrm{~V}}^{*}=\frac{\mathrm{s}}{2\left(1+\mathrm{v}_{\mathrm{s}}\right)},
$$

where $v_{\mathrm{s}}$ is the solid phase Poisson's ratio. Now deformation of joints cannot be neglected, due to the presence of $v_{\mathrm{s}}$, which is restricted to the interval $[-1,1]$. It is obvious that the strain field inside the cell of the regular lattice would be fully uniform for $\Sigma^{\mathrm{k}}$ macroload only if $\mathrm{v}_{\mathrm{s}}=-1$ giving $\mathrm{k}_{\mathrm{V}}^{*}=\mathrm{s} / 4$ and for $\Sigma^{1 G}$ macroload only if $v_{\mathrm{s}}=1$ yielding $\mathrm{G}_{1, \mathrm{~V}}^{*}=\mathrm{s} / 4$, which are the upper bounds on the properties of $2 \mathrm{D}$ cellular media.

\section{References}

[1] Allaire G., Aubry S.: "On Optimal Microstructures for a Plane Shape Optimization Problem.” Struct. Opt. Vol. 17 (1999), p. 86-94.

[2] Allaire G., Kohn R. V.: “Optimal Design for Minimum Weight and Compliance in Plane Stress Using Extremal Microstructures.” Eur. J. Mech., A/Solids, Vol. 12 (1993), p. 839-878.

[3] Avellaneda M.: "Optimal Bounds and Microgeometries for Elastic Two-Phase Composites." J. Appl. Math., SIAM, Vol. 47 (1987), p. 1216-1228.

[4] Bakhvalov N., Panasenko G.: "Homogenization: Averaging Processes in Periodic Media.” Dordrecht, Boston, London: Kluwer Academic Publishers, 1989.

[5] Bensoussan A., Lions J. L., Papanicolau G.: "Asymptotic Analysis for Periodic Structures.” North Holland, Amsterdam, 1978.

[6] Christensen R. M.: "Heterogeneous Material Mechanics at Various Scales." Appl. Mech. Rev., Vol. 47 (1994), S20-S33.
[7] Christensen R. M.: "The Hierarchy of Microstructures for Low Density Materials.” Z. Angew. Math. Phys., Vol. 46 (1995), p. S507-S521.

[8] Dimitrovová Z.: "Effective Constitutive Properties of Linear Elastic Cellular Solids with Randomly Oriented Cells.” J. Appl. Mech., ASME, Vol. 66 (1999), p. 918-915.

[9] Dimitrovová Z., Faria L.: "New Methodology to Establish Bounds on Effective Properties of Cellular Solids." J. Mech. Compos. Mater. Struct., Vol. 6 (1999), p. 331-346.

[10] Duvaut G.: "Homogenisation et Materiaux Composites." In: "Theoretical and Applied Mechanics." Ed. P. Ciarlet; M. Rouseau, Amsterdam, North-Holland, 1976.

[11] Gibson L. J., Ashby M. F.: "Cellular Solids. Structure and Properties.” Pergamon Press, Oxford, 1988.

[12] Gibianski L. V., Sigmund O.: "Multiphase Elastic Composites with Extremal Bulk Modulus." J. Mech. Phys. Solids, Vol. 48 (2000), p. 461-498.

[13] Grenestedt J. L.: "Effective Elastic Behavior of Some Models for 'Perfect' Cellular Solids." Int. J. Solids Struct., Vol. 36 (1999), p. 1471-1501.

[14] Guedes J. M., Rodrigues H. C., Bendsoe M. P.: “A Material Optimization Model to Approximate Energy Bounds for Cellular Materials under Multiload Conditions." Struct. Opt., Vol. 25 (2003), p. 446-452.

[15] Hashin Z.: "The Elastic Moduli of Heterogeneous Materials.” J. Appl. Mech., ASME, Vol. 29 (1962), p. 143-150.

[16] Hashin Z.: "Theory of Composite Materials." In: "Mechanics of Composite Materials." Ed. F. W. Wendt, H. Liebowitz, N. Perrone. Pergamon Press, Oxford, (1970).

[17] Hashin Z.: "Analysis of Composite Materials-A Survey." J. Appl. Mech., ASME, Vol. 50 (1983), p. 481-505.

[18] Hashin Z., Shtrikman S.: "A Variational Approach to the Theory of the Elastic Behavior of Polycrystals." J. Mech. Phys. Solids, Vol. 10 (1962), p. 343-352. 
[19] Hashin Z., Shtrikman S.: "A Variational Approach to the Theory of the Elastic Behavior of Multiphase Materials." J. Mech. Phys. Solids, Vol. 11 (1963), p. 127-140.

[20] Hill R.: "Elastic Properties of Reinforced Solids: Some Theoretical Principles." J. Mech. Phys. Solids, Vol. 11 (1963), p. 357-372.

[21] Kraynik A. M., Warren W. E.: "The Elastic Behavior of Low-Density Cellular Plastics." In: "Low Density Cellular Plastics: Physical Basis of Behavior." Ed. N. C. Hilyard, A. Cunningham. Chapman \& Hall, London, 1994, p. 217.

[22] Lekhnitskii S. G.: "Theory of Elasticity of an Anisotropic Body.” Mir Publishers, Moscow, 1981.

[23] Li K., Gao X.-L., Roy A. K.: "Micromechanics Model for Three-dimensional Open-cell Foams Using a Tetrakaidecahedral Unit Cell and Castigliano's Second Theorem." Compos. Sci. Techn., Vol. 63 (2003), p.1769-1781.

[24] Nemat-Nasser S., Hori M.: "Micromechanics: Overall Properties of Heterogeneous Materials." North-Holland Series in Applied Mathematics and Mechanics, Vol. 37, ed. J. Achenbach, B. Budiansky, H.A. Lauwerier, P.G. Saffman, L. Van Wijngaarden, J.R. Willis, North-Holland-Amsterdam, London, New York, Tokyo, 1993.

[25] Neves M. M, Guedes J. M., Rodrigues H.: "Optimal Design of Periodic Linear Elastic Microstructures." Comput. \& Struct., Vol. 76 (2000), p. 421-429.

[26] Noor A. K.: "Continuum Modeling for Repetitive Lattice Structures.” Appl. Mech. Rev., Vol. 41 (1988), p. 285-296.
[27] Sigmund O.: "Materials with Prescribed Constitutive Parameters: an Inverse Homogenization Problem." Int. J. Solids Struct., Vol. 31 (1994), p. 2313-2329.

[28] Suquet P. M.: "Elements of Homogenization for Inelastic Solid Mechanics." In: "Homogenization Techniques for Composite Media." Ed. E. Sanchez-Palencia, A. Zaoui, Lecture Notes in Physics, 272, Springer-Verlag, 1985, p. 193-278.

[29] Warren W. E., Kraynik A. M.: "The Linear Elastic Properties of Open-Cell Foams.” J. Appl. Mech., ASME, Vol. 55 (1988), p. 341-346.

[30] Warren W. E., Kraynik A. M.: "Linear Elastic Behavior of a Low-Density Kelvin Foam with Open Cells.” J. Appl. Mech., ASME, Vol. 64 (1997), p. 787-794.

[31] Zhu H. X., Knott J. F., Mills N. J.: "Analysis of the Elastic Properties of Open-Cell Foams with Tetrakaidecahedral Cells." J. Mech. Phys. Solids, Vol. 45 (1997), p. 319-343.

Zuzana Dimitrovová

phone: +351218419462

fax: +351218417915

e-mail: zdimitro@dem.ist.utl.pt / zuzana@dem.isel.ipl.pt

DEM / ISEL

IDMEC / IST

Instituto Superior Técnico

Av. Rovisco pais, 1

1049-001 Lisbon, Portugal 